\title{
Cross-Immunity-Induced Backward Bifurcation for a Model of Transmission Dynamics of Two Strains of Influenza
}

\author{
October 6, 2012
}

S.M. Garba ${ }^{\ddagger}$, M.A. Safi $^{\S}$ and A.B. Gumel ${ }^{\dagger, 1}$

${ }^{\dagger}$ Department of Mathematics, University of Manitoba, Winnipeg, Manitoba, R3T 2N2, Canada.

$\ddagger$ Department of Mathematics and Applied Mathematics, University of Pretoria, Pretoria 0002, South Africa.

$\S$ Department of Mathematics, The Hashemite University, Zarqa, Jordan.

\begin{abstract}
A new deterministic model for the transmission dynamics of two strains of influenza is designed and used to qualitatively assess the role of cross-immunity on the transmission process. It is shown that incomplete cross-immunity could induce the phenomenon of backward bifurcation when the associated reproduction number is less than unity. The model undergoes competitive exclusion (where Strain $i$ drives out Strain $j$ to extinction whenever $\mathcal{R}_{0 i}>1>\mathcal{R}_{0 j} ; i, j=$ $1,2, i \neq j)$. For the case where infection with one strain confers complete immunity against infection with the other strain, it is shown that the disease-free equilibrium of the model is globally-asymptotically stable whenever the reproduction number is less than unity. In the absence of cross-immunity, the model can have a continuum of co-existence endemic equilibria (which is shown to be globally-asymptotically stable for a special case). When infection with one strain confers incomplete immunity against the other. Numerical simulations of the model show that the two strains co-exist, with Strain $i$ dominating (but not driving out Strain $j$ ), whenever $\mathcal{R}_{0 i}>\mathcal{R}_{0 j}>1$.
\end{abstract}

Keywords: cross-immunity; multiple strains; equilibria; co-existence; stability.

${ }^{1}$ Corresponding author. Email: gumelab@cc.umanitoba.ca 


\section{Introduction}

One of the important problems in mathematical epidemiology is the study of the transmission dynamics of diseases with multiple strains in the presence of partial or complete immunity. Consequently, the mathematical modeling of diseases with multiple pathogen strains, such as dengue fever, HIV/AIDS, influenza, malaria and West Nile virus, has received considerable attention (see, for instance, [1, 2, 12, 15, 19, 27, 29, 23, $26,34,39]$ and some of the references therein). These studies have, in general, focussed on the determination of threshold condition(s) for the co-existence of the strains, as well as the evaluation of the role of cross-immunity (defined as a scenario where infection with one strain confers partial or complete protection against infection with another strain) in the transmission dynamics of the disease strains.

In a multi-strain dynamics situation, infection by one or more of the strains may modify the sensitivity to infection by the other strains [15, 23, 26, 29, 38]. Some of the main questions of epidemiological interest, in studies of modeling multi-strain dynamics, are:

(i) which strain(s) will dominate in the long run (i.e., does competitive exclusion phenomenon occurs)?

(ii) under what conditions will the strains co-exist?

(iii) what is the effect of cross-immunity (partial or complete) on the multi-strain dynamics?

These questions could be addressed using the threshold quantity known as the basic reproduction number of the disease $[3,4,27]$, which represents the average number of secondary cases generated by a typical infected individual in a completely susceptible population. Past research has estimated that the reproduction number of the 19181919 influenza pandemic and other seasonal strains of influenza ranged between 1.5 and $5.4[15,16,17,18,21,26,35,36]$.

Using an SIQR deterministic model for the dynamics of two strains of influenza in the presence of isolation of symptomatic cases, Nuno et al. [29] showed that crossimmunity and host isolation could induce sustained periodic oscillations. Bremmerman and Thieme [8] shows the phenomenon of competitive exclusion (where the strain with the largest reproduction number persists and eliminates the remaining strains) in a simple model with multiple strains (similar results were obtained in $[11,20,23]$ ). Gumel [26] shows co-existence of two strains (avian and mutant) of influenza when their reproduction numbers exceed unity using avian-human model in the presence of isolation (system does not undergo competitive exclusion).

The aim of this study is to rigorously assess the role of cross-immunity on the transmission dynamics of two strains of influenza in a population. To achieve this objective, a new deterministic model (which extends the model in [29]) will be designed. 
The paper is organized as follows. The extended model is formulated in Section 2 and analysed in Section 3.

\section{Formulation of Mathematical Model}

The model is based on the transmission dynamics of two strains of influenza. The total population at time $t$, denoted by $N(t)$, is subdivided into susceptible $(S(t))$; exposed to strain $i(i=1,2)\left(E_{i}\right)$; infectious with strain $i\left(I_{i}\right)$; recovered from strain $i\left(R_{i}\right)$; recovered from strain $i$ and exposed to strain $j(i, j=1,2 i \neq j)\left(E_{i j}\right)$; recovered from strain $i$ and infectious with strain $j\left(I_{i j}\right)$; and individuals recovered from infection with both strains $(M)$, so that

$N(t)=S(t)+E_{1}(t)+I_{1}(t)+R_{1}(t)+E_{2}(t)+I_{2}(t)+R_{2}(t)+E_{12}(t)+I_{12}(t)+E_{21}(t)+I_{21}(t)+M(t)$.

The model to be considered is given by the following deterministic system of non-linear differential equations (where a dot represents differentiation with respect to $t$; and all associated parameters are non-negative for all $t \geq 0$ ):

$$
\begin{aligned}
\dot{S} & =\Pi+\xi M-\frac{\beta_{1} S\left(\eta_{1} E_{1}+I_{1}\right)}{N}-\frac{\beta_{2} S\left(\eta_{2} E_{2}+I_{2}\right)}{N}-\frac{\beta_{12} S\left(\eta_{12} E_{12}+I_{12}\right)}{N} \\
& -\frac{\beta_{21} S\left(\eta_{21} E_{21}+I_{21}\right)}{N}-\mu S, \\
\dot{E}_{1} & =\frac{\beta_{1}\left(\eta_{1} E_{1}+I_{1}\right)}{N} S+\frac{\beta_{21}\left(\eta_{21} E_{21}+I_{21}\right)}{N} S-\sigma_{1} E_{1}-\mu E_{1}, \\
\dot{I}_{1} & =\sigma_{1} E_{1}-\gamma_{1} I_{1}-\mu I_{1}-\delta_{1} I_{1}, \\
\dot{R_{1}} & =\gamma_{1} I_{1}-\theta_{2} \frac{\beta_{2} R_{1}\left(\eta_{2} E_{2}+I_{2}\right)}{N}-\theta_{2} \frac{\beta_{12} R_{1}\left(\eta_{12} E_{12}+I_{12}\right)}{N}-\mu R_{1}, \\
\dot{E_{2}} & =\frac{\beta_{2}\left(\eta_{2} E_{2}+I_{2}\right)}{N} S+\frac{\beta_{12}\left(\eta_{12} E_{12}+I_{12}\right)}{N} S-\sigma_{2} E_{2}-\mu E_{2}, \\
\dot{I_{2}} & =\sigma_{2} E_{2}-\gamma_{2} I_{2}-\mu I_{2}-\delta_{2} I_{2}, \\
\dot{R}_{2} & =\gamma_{2} I_{2}-\theta_{1} \frac{\beta_{1} R_{2}\left(\eta_{1} E_{1}+I_{1}\right)}{N}-\theta_{1} \frac{\beta_{21} R_{2}\left(\eta_{21} E_{21}+I_{21}\right)}{N}-\mu R_{2}, \\
\dot{E_{12}} & =\theta_{2} \frac{\beta_{2} R_{1}\left(\eta_{2} E_{2}+I_{2}\right)}{N}+\theta_{2} \frac{\beta_{12} R_{1}\left(\eta_{12} E_{12}+I_{12}\right)}{N}-\sigma_{12} E_{12}-\mu E_{12}, \\
\dot{I_{12}} & =\sigma_{12} E_{12}-\gamma_{12} I_{12}-\mu I_{12}-\delta_{12} I_{12}, \\
\dot{E_{21}} & =\theta_{1} \frac{\beta_{1} R_{2}\left(\eta_{1} E_{1}+I_{1}\right)}{N}+\theta_{1} \frac{\beta_{21} R_{2}\left(\eta_{21} E_{21}+I_{21}\right)}{N}-\sigma_{21} E_{21}-\mu E_{21}, \\
\dot{I_{21}} & =\sigma_{21} E_{21}-\gamma_{21} I_{21}-\mu I_{21}-\delta_{21} I_{21}, \\
\dot{M} & =\gamma_{12} I_{12}+\gamma_{21} I_{21}-\xi M-\mu M,
\end{aligned}
$$

where $\Pi$ is the recruitment rate into the community, $\xi$ is the rate of loss of natural immunity by recovered individuals, $\beta_{i}$ (where $i=1,2$ here and elsewhere below) is the infection rate with strain $i, \beta_{i j}(i, j=1,2 ; i \neq j)$ represents the transmission rate for 
individuals who recovered from strain $i$ but exposed to strain $j$ and $\mu$ is the natural death rate. The modification parameters $\eta_{i}<1$ accounts for the assumed reduction of exposed individuals (those in the $E_{i}$ classes) in relation to infectious individuals (in the $I_{i}$ classes); the parameters $\eta_{i j}$ are similarly defined.

Furthermore, $\sigma_{i}$ is the transition rate of individuals exposed with strain $i$ (i.e., those in the $E_{i}$ class) to the corresponding infectious $\left(I_{i}\right)$ class (the parameters $\sigma_{i j}$ are similarly defined). The parameters $\gamma_{i}$ and $\delta_{i}$ represent, respectively, the recovery rates and disease-induced death rate of individuals infected with strain $i$ (the parameters $\gamma_{i j}$ and $\delta_{i j}$ are defined similarly). The modification parameters $0 \leq \theta_{i} \leq 1$ account for the assumed reduction of susceptibility to strain $j$ of individuals who recovered from strain $i$ (i.e., $0 \leq \theta_{i} \leq 1$ ) captures the cross-immunity of individuals who recovered from one strain against acquiring infection with the other). These parameters are described in Table 1, and a flow diagram of the model is depicted in Figure 1.

The model (1) is an extension of the two-strain influenza model developed by Nuno et al. [29], by

(i) adding epidemiological compartments for exposed individuals ( $E_{1}, E_{2}, E_{12}$ and $E_{21}$ ). The incubation period of influenza is 1-4 days (average is about 2 days) $[14]$;

(ii) allowing for disease transmission by exposed individuals (at reduced rates $\beta_{i} \eta_{i}$ for those with primary infection; and $\beta_{i j} \eta_{i j}$ for those with secondary infection). Clinical evidence suggests that individuals infected with an influenza virus can transmit infection even before the onset of symptoms (typically a day before symptoms develop) [14];

(iii) allowing heterogeneity in transmission due to recovery from infection with one strain (i.e., individuals in the $E_{i j}$ and $I_{i j}$ classes transmit infection at different rates in comparison to those in the corresponding $E_{i}$ and $I_{i}$ classes). Same rate $\left(\beta_{i}\right)$ is used in Nuno et al. [29], for disease transmission by individuals infected with strain $i$ (regardless of whether or not the infected individuals recover from strain $j$ ). Clinical studies show differential heterogeneity in disease transmission by individuals previously infected with different disease strain $[9,17,25]$;

(iv) allow for the loss of natural immunity in individuals who recovered from both strains (at the rate $\xi$ ) [6].

It should be noted that, unlike in [29], the model (1) does not incorporate the isolation of symptomatic cases (i.e., while isolation-adjusted incidence is used to model the infection rate in [29], standard incidence function is used in (1); the reader may refer to [15] for the disadvantages associated with implementing isolation strategy, including the difficulty of detecting infected individuals and the cost of isolation in the context 
of influenza). Furthermore, the model (1) can also be used for any influenza-like illness (where exposed individuals can transmit infection) with two strains. In addition to the above extensions, rigorous qualitative analysis will be provided for the resulting model.

\subsection{Basic Properties of the Model}

For the model (1) to be epidemiologically meaningful, it is important to prove that all its state variables are non-negative for all time $(t)$. In other words, the solutions of the model (1) with positive initial data will remain positive for all $t \geq 0$. The following result can be proven (see, for instance, $[8,22,24,37,40]$ )

Theorem 1 Let the initial data $S(0)>0, E_{1}(0)>0, I_{1}(0)>0, R_{1}(0)>0, E_{2}(0)>$ $0, I_{2}(0)>0, R_{2}(0)>0, E_{12}(0)>0, I_{12}(0)>0, E_{21}(0)>0, I_{21}(0)>0, M(0)>0$, then the solutions $S, E_{1}, I_{1}, R_{1}, E_{2}, I_{2}, R_{2}, E_{12}, I_{12}, E_{21}, I_{21}, M$ of the model (1) are positive for all $t \geq 0$. Furthermore,

$$
\limsup _{t \rightarrow \infty} N(t) \leq \frac{\Pi}{\mu}
$$

\subsubsection{Invariant region}

\section{Lemma 1 The region}

$$
\begin{aligned}
\Omega= & \left\{\left(S, E_{1}, I_{1}, R_{1}, E_{2}, I_{2}, R_{2}, E_{12}, I_{12}, E_{21}, I_{21}, M\right) \in \mathbb{R}_{+}^{12}:\right. \\
& \left.S+E_{1}+I_{1}+R_{1}+E_{2}+I_{2}+R_{2}+E_{12}+I_{12}+E_{21}+I_{21}+M \leq \frac{\Pi}{\mu}\right\}
\end{aligned}
$$

is positively-invariant for the model (1).

Proof. Adding the equations in the model system (1) gives

$$
\frac{d N(t)}{d t}=\Pi-\mu N(t)-\left(\delta_{1} I_{1}+\delta_{2} I_{2}+\delta_{12} I_{12}+\delta_{21} I_{21}\right) .
$$

It follows from (2) that

$$
\frac{d N(t)}{d t} \leq \Pi-\mu N(t)
$$

Thus (using standard comparison theorem [32]), $N(t) \leq N(0) \mathrm{e}^{-\mu(\mathrm{t})}+\frac{\Pi}{\mu}\left[1-\mathrm{e}^{-\mu(\mathrm{t})}\right]$. In particular, $N(t) \leq \Pi / \mu$. Thus, $\Omega$ is positively-invariant. Hence, it is sufficient to consider the dynamics of the model (1) in $\Omega$. In this region, the model can be considered as been epidemiologically and mathematically well-posed [27]. 


\subsection{Stability of Disease-Free Equilibria (DFE)}

\subsubsection{Local stability}

The DFE of the model (1) is given by

$$
\begin{aligned}
\mathcal{E}_{0} & =\left(S^{*}, E_{1}^{*}, I_{1}^{*}, R_{1}^{*}, E_{2}^{*}, I_{2}^{*}, R_{2}^{*}, E_{12}^{*}, I_{12}^{*}, E_{21}^{*}, I_{21}^{*}, M^{*}\right), \\
& =\left(\frac{\Pi}{\mu}, 0,0,0,0,0,0,0,0,0,0,0\right) .
\end{aligned}
$$

The linear stability of $\mathcal{E}_{0}$ can be established using the next generation operator method on system (1). Using the notation in [41], the matrices $F$ (for the new infection terms) and $V$ (of the transition terms) are given, respectively, by

$$
\begin{aligned}
F & =\left[\begin{array}{cccccccc}
\beta_{1} \eta_{1} & \beta_{1} & 0 & 0 & 0 & 0 & \beta_{21} \eta_{21} & \beta_{21} \\
0 & 0 & 0 & 0 & 0 & 0 & 0 & 0 \\
0 & 0 & \beta_{2} \eta_{2} & \beta_{2} & \beta_{12} \eta_{12} & \beta_{12} & 0 & 0 \\
0 & 0 & 0 & 0 & 0 & 0 & 0 & 0 \\
0 & 0 & 0 & 0 & 0 & 0 & 0 & 0 \\
0 & 0 & 0 & 0 & 0 & 0 & 0 & 0 \\
0 & 0 & 0 & 0 & 0 & 0 & 0 & 0 \\
0 & 0 & 0 & 0 & 0 & 0 & 0 & 0
\end{array}\right], \\
V & =\left[\begin{array}{cccccccc}
K_{1} & 0 & 0 & 0 & 0 & 0 & 0 & 0 \\
-\sigma & K_{2} & 0 & 0 & 0 & 0 & 0 & 0 \\
0 & 0 & K_{3} & 0 & 0 & 0 & 0 & 0 \\
0 & 0 & -\sigma_{2} & K_{4} & 0 & 0 & 0 & 0 \\
0 & 0 & 0 & 0 & K_{5} & 0 & 0 & 0 \\
0 & 0 & 0 & 0 & -\sigma_{12} & K_{6} & 0 & 0 \\
0 & 0 & 0 & 0 & 0 & 0 & K_{7} & 0 \\
0 & 0 & 0 & 0 & 0 & 0 & -\sigma_{21} & K_{8}
\end{array}\right],
\end{aligned}
$$

where, $K_{1}=\mu+\sigma_{1}, K_{2}=\mu+\gamma_{1}+\delta_{1}, K_{3}=\mu+\sigma_{2}, K_{4}=\mu+\gamma_{2}+\delta_{2}, K_{5}=$ $\mu+\sigma_{12}, K_{6}=\mu+\gamma_{12}+\delta_{12}, K_{7}=\mu+\sigma_{21}$ and $K_{8}=\mu+\gamma_{21}+\delta_{21}$.

It follows that the basic reproduction number of the model (1), denoted by $\mathcal{R}_{0}$, is given by (where $\rho$ is the spectral radius)

$$
\mathcal{R}_{0}=\rho\left(F V^{-1}\right)=\max \left\{\mathcal{R}_{01}, \mathcal{R}_{02}\right\}
$$

where $\mathcal{R}_{01}$ and $\mathcal{R}_{02}$ are the associated reproduction numbers for strain 1 and strain 2 , respectively, given by 


$$
\mathcal{R}_{01}=\frac{\beta_{1}\left(\eta_{1} K_{2}+\sigma_{1}\right)}{K_{1} K_{2}} \text { and } \mathcal{R}_{02}=\frac{\beta_{2}\left(\eta_{2} K_{4}+\sigma_{2}\right)}{K_{3} K_{4}} .
$$

Lemma 2 The DFE, $\mathcal{E}_{0}$, of the model (1) is locally-asymptotically stable (LAS) if $\mathcal{R}_{0}<1$, and unstable if $\mathcal{R}_{0}>1$.

The threshold quantity, $\mathcal{R}_{0}=\max \left\{\mathcal{R}_{01}, \mathcal{R}_{02}\right\}$, is the basic reproduction number of the disease $[3,4,27]$. It represents the average number of secondary cases generated by a typical infected individual in a completely susceptible population. The epidemiological implication of Lemma 2 is that when $\mathcal{R}_{0}$ is less than unity, a small influx of infected individuals into the community would not generate large outbreaks, and the disease dies out in time.

\subsubsection{Backward bifurcation analysis}

It is instructive to characterize the types of bifurcation the model (1) may undergo. We claim the following result (the proof is given in Appendix A):

Theorem 2 The model (1) exhibits backward bifurcation at $\mathcal{R}_{0}=1$ whenever a bifurcation coefficient, denoted by a (and given by (32)), is positive.

The public health implication of the backward bifurcation phenomenon of the model (1) is that the classical epidemiological requirement of having the reproduction number $\left(\mathcal{R}_{0}\right)$ to be less than unity, while necessary, is no longer sufficient for the effective control of the disease. In other words, the backward bifurcation property of the model (1) makes effective diseases control difficult. It is worth stating that, setting $\theta_{1}=\theta_{2}=0$ in the inequality (32) gives (noting that all parameters of the model are positive and $\beta_{1}^{*}>0$ is given in Appendix A):

$$
a=-\frac{4 \mu}{\Pi}\left[\frac{\beta_{1}^{*}\left(\eta_{1} K_{2}+\sigma_{1}\right)}{\sigma_{1}}+\frac{\beta_{2}\left(\eta_{2} K_{4}+\sigma_{2}\right)}{\sigma_{2}}\right]\left(2+\frac{K_{2}}{\sigma_{1}}+\frac{K_{4}}{\sigma_{2}}+\frac{\gamma_{1}+\gamma_{2}}{\mu}\right)<0 .
$$

Thus, it follows from Theorem 4.5 in [13] that the model (1) does not undergo backward bifurcation in this setting. Hence, this study shows that the backward bifurcation phenomenon of the model (1) is caused by the incomplete cross-immunity property of the model $\left(0<\theta_{1}, \theta_{2}<1\right)$. A global asymptotic stability result is established below for the DFE of the model (to completely rule out backward bifurcation for the case when infection with one strain confers complete cross-immunity against the other strain). It is worth stating that the phenomenon of backward bifurcation is not rigorously established in the study by Nuno et al. [29] (the phenomenon was shown using numerical simulations). This, to the authors' knowledge, is the first time it is rigorously shown that incomplete cross-immunity could induce backward bifurcation in the transmission dynamics of two strains of influenza. Garba and Gumel [23] also established similar result in the dynamics of two strains of dengue fever. 


\subsection{Global Stability of DFE: Special Case $\theta_{1}=\theta_{2}=0$}

Consider the model (1), for the case when infection with one strain confers complete immunity against infection with the other strain (i.e., $\theta_{1}=\theta_{2}=0$ ). It follows, by setting $\theta_{1}=\theta_{2}=0$ in (1), that $E_{12} \rightarrow 0, I_{12} \rightarrow 0, E_{21} \rightarrow 0, I_{21} \rightarrow 0$ and $M \rightarrow 0$ as $t \rightarrow \infty$. Thus, the last five equations of the model decouple, so that the model (1) reduces to:

$$
\begin{aligned}
\dot{S} & =\Pi-\lambda_{1} S-\lambda_{2} S-\mu S, \\
\dot{E}_{1} & =\lambda_{1} S-K_{1} E_{1}, \\
\dot{I}_{1} & =\sigma_{1} E_{1}-K_{2} I_{1} \\
\dot{R}_{1} & =\gamma_{1} I_{1}-\mu R_{1} \\
\dot{E}_{2} & =\lambda_{2} S-K_{3} E_{2} \\
\dot{I}_{2} & =\sigma_{2} E_{2}-K_{4} I_{2} \\
\dot{R}_{2} & =\gamma_{2} I_{2}-\mu R_{2} .
\end{aligned}
$$

where, now, $\lambda_{1}=\frac{\beta_{1}\left(\eta_{1} E_{1}+I_{1}\right)}{N}$ and $\lambda_{2}=\frac{\beta_{2}\left(\eta_{2} E_{2}+I_{2}\right)}{N}$.

The dynamics of the reduced model (7) will be considered in the following invariant region

$$
\Gamma=\left\{\left(S, E_{1}, I_{1}, R_{1}, E_{2}, I_{2}, R_{2}\right) \in \mathbb{R}_{+}^{7}: N \leq \frac{\Pi}{\mu}\right\}
$$

The DFE of the reduced model is given by $\mathcal{E}_{0}^{r}=\left(S, E_{1}, I_{1}, R_{1}, E_{2}, I_{2}, R_{2}\right)=\left(\frac{\Pi}{\mu}, 0,0,0,0,0,0\right)$. The model (7) has the same reproduction number, $\mathcal{R}_{0}=\max \left\{\mathcal{R}_{01}, \mathcal{R}_{02}\right\}$, as model (1).

Theorem 3 The DFE, $\mathcal{E}_{0}^{r}$, of the reduced model (7) is GAS in $\Gamma$ whenever $\mathcal{R}_{0} \leq 1$.

Proof. Consider the Lyapunov function for the model (7):

$$
\mathcal{F}=\left(\frac{\eta_{1} K_{2}+\sigma_{1}}{K_{1}}\right) E_{1}+I_{1}+\left(\frac{\eta_{2} K_{4}+\sigma_{2}}{K_{3}}\right) E_{2}+I_{2},
$$

with Lyapunov derivative given by 


$$
\begin{aligned}
\dot{\mathcal{F}} & =\left(\frac{\eta_{1} K_{2}+\sigma_{1}}{K_{1}}\right) \dot{E}_{1}+\dot{I}_{1}+\left(\frac{\eta_{2} K_{4}+\sigma_{2}}{K_{3}}\right) \dot{E}_{2}+\dot{I}_{2}, \\
& =\left(\frac{\eta_{1} K_{2}+\sigma_{1}}{K_{1}}\right)\left[\frac{\beta_{1}\left(\eta_{1} E_{1}+I_{1}\right)}{N} S-K_{1} E_{1}\right]+\sigma_{1} E_{1}-K_{2} I_{1} \\
& +\left(\frac{\eta_{2} K_{4}+\sigma_{2}}{K_{3}}\right)\left[\frac{\beta_{2}\left(\eta_{2} E_{2}+I_{2}\right)}{N} S-K_{3} E_{2}\right]+\sigma_{2} E_{2}-K_{4} I_{2}, \\
& \leq\left(\frac{\eta_{1} K_{2}+\sigma_{1}}{K_{1}}\right)\left[\beta_{1}\left(\eta_{1} E_{1}+I_{1}\right)-K_{1} E_{1}\right]+\sigma_{1} E_{1}-K_{2} I_{1} \\
& +\left(\frac{\eta_{2} K_{4}+\sigma_{2}}{K_{3}}\right)\left[\beta_{2}\left(\eta_{2} E_{2}+I_{2}\right)-K_{3} E_{2}\right]+\sigma_{2} E_{2}-K_{4} I_{2} \text { since } S \leq N \text { in } \Gamma \\
& =\beta_{1}\left(\frac{\eta_{1} K_{2}+\sigma_{1}}{K_{1}}\right)\left(\eta_{1} E_{1}+I_{1}\right)-\left(\eta_{1} K_{2}+\sigma_{1}\right) E_{1}+\sigma_{1} E_{1}-K_{2} I_{1} \\
& +\beta_{2}\left(\frac{\eta_{2} K_{4}+\sigma_{2}}{K_{3}}\right)\left(\eta_{2} E_{2}+I_{2}\right)-\left(\eta_{2} K_{4}+\sigma_{2}\right) E_{2}+\sigma_{2} E_{2}-K_{4} I_{2}, \\
& =K_{2}\left(\eta_{1} E_{1}+I_{1}\right)\left(\mathcal{R}_{01}-1\right)+K_{4}\left(\eta_{2} E_{2}+I_{2}\right)\left(\mathcal{R}_{02}-1\right) . \\
& =K_{2} \mathcal{R}_{01}\left(\eta_{1} E_{1}+I_{1}\right)-\eta_{1} K_{2} E_{1}-K_{2} I_{1}+K_{4} \mathcal{R}_{02}\left(\eta_{2} E_{2}+I_{2}\right)-\eta_{2} K_{4} E_{2}-K_{4} I_{2}, \\
&
\end{aligned}
$$

Thus $\dot{\mathcal{F}} \leq 0$ if $\mathcal{R}_{0}=\max \left\{\mathcal{R}_{01}, \mathcal{R}_{02}\right\} \leq 1$ with $\dot{\mathcal{F}}=0$ if $E_{1}=I_{1}=E_{2}=I_{2}=0$. Substituting $E_{1}=I_{1}=E_{2}=I_{2}=0$ in the first equation in (7) shows that $S(t) \rightarrow \Pi / \mu$ as $t \rightarrow \infty$. Similarly, substituting $E_{1}=I_{1}=E_{2}=I_{2}=0$ in the equations for $R_{1}$ and $R_{2}$ show that $\left(R_{1}, R_{2}\right) \rightarrow(0,0)$ as $t \rightarrow \infty$. Further, the largest compact invariant set in $\left\{\left(S, E_{1}, I_{1}, R_{1}, E_{2}, I_{2}, R_{2}\right) \in \Gamma: d \mathcal{F} / d t=0\right\}$ is the singleton $\left\{\mathcal{E}_{0}^{r}\right\}$. It follows, from the LaSalle's Invariance Principle [33], that every solution to the equations in (7) with initial conditions in $\Gamma$ converges to the DFE $\mathcal{E}_{0}^{r}$ as $t \rightarrow \infty$.

This result shows that, for the case when infection with one strain confers complete immunity against the other strain, the DFE of the model (1) is GAS (hence, the disease (i.e., both strains) will be eliminated from the community if $\mathcal{R}_{0}<1$ in this case). It should be mentioned that such global-asymptotic result was not established for the 
DFE of the model presented by Nuno et al. [29].

\section{$3 \quad$ Existence and Stability of Boundary Equilibria}

The approach in $[23,31]$ will be used to explore the possibility of the existence and stability of the positive equilibria (i.e., equilibria where at least one of the infected variables of the model (1) is non-zero). Let

$$
\mathcal{E}_{12}=\left(S^{* *}, E_{1}^{* *}, I_{1}^{* *}, R_{1}^{* *}, E_{2}^{* *}, I_{2}^{* *}, R_{2}^{* *}, E_{12}^{* *}, I_{12}^{* *}, R_{12}^{* *}, E_{21}^{* *}, I_{21}^{* *}, M^{* *}\right)
$$

represents any arbitrary equilibrium of the model (1). Further, let

$\lambda_{1}^{* *}=\frac{\beta_{1}\left(\eta_{1} E_{1}^{* *}+I_{1}^{* *}\right)+\beta_{21}\left(\eta_{21} E_{21}^{* *}+I_{21}^{* *}\right)}{N^{* *}}$ and $\lambda_{2}^{* *}=\frac{\beta_{2}\left(\eta_{2} E_{2}^{* *}+I_{2}^{* *}\right)+\beta_{12}\left(\eta_{12} E_{12}^{* *}+I_{12}^{* *}\right)}{N^{* *}}(10)$

be the forces of infection of strains 1 and 2, respectively, at endemic steady-state (where $N^{* *}$ is the total population size at the endemic steady-state). Solving the equations in (1) at endemic steady-state gives

$$
\begin{aligned}
S^{* *} & =\frac{\Pi}{A_{1}}, E_{1}^{* *}=\frac{\Pi \lambda_{1}^{* *}}{K_{1} A_{1}}, I_{1}^{* *}=\frac{\Pi \lambda_{1}^{* *} \sigma_{1}}{K_{1} K_{2} A_{1}}, R_{1}^{* *}=\frac{\Pi \lambda_{1}^{* *} \sigma_{1} \gamma_{1}}{K_{1} K_{2} A_{1} A_{3}}, \\
E_{2}^{* *} & =\frac{\Pi \lambda_{2}^{* *}}{K_{3} A_{1}}, I_{2}^{* *}=\frac{\Pi \lambda_{2}^{* *} \sigma_{2}}{K_{3} K_{4} A_{1}}, R_{2}^{* *}=\frac{\Pi \lambda_{2}^{* *} \sigma_{2} \gamma_{2}}{K_{3} K_{4} A_{1} A_{2}}, E_{12}^{* *}=\frac{\Pi \lambda_{1}^{* *} \lambda_{2}^{* *} \sigma_{1} \gamma_{1} \theta_{2}}{K_{1} K_{2} K_{5} A_{1} A_{3}}, \\
I_{12}^{* *} & =\frac{\Pi \lambda_{1}^{* *} \lambda_{2}^{* *} \sigma_{1} \sigma_{3} \gamma_{1} \theta_{2}}{K_{1} K_{2} K_{5} K_{6} A_{1} A_{3}}, E_{21}^{* *}=\frac{\Pi \lambda_{1}^{* *} \lambda_{2}^{* *} \sigma_{2} \gamma_{2} \theta_{1}}{K_{3} K_{4} K_{7} A_{1} A_{2}}, I_{21}^{* *}=\frac{\Pi \lambda_{1}^{* *} \lambda_{2}^{* *} \sigma_{2} \sigma_{4} \gamma_{2} \theta_{1}}{K_{3} K_{4} K_{7} K_{8} A_{1} A_{2}}, \\
M^{* *} & =\frac{\Pi \lambda_{1}^{* *} \lambda_{2}^{* *} \sigma_{2} \theta_{1}\left(\gamma_{3} K_{8}+\gamma_{4} \sigma_{4}\right)}{K_{3} K_{4} K_{7} K_{8} K_{9} A_{1} A_{2}},
\end{aligned}
$$

with $A_{1}=\lambda_{1}^{* *}+\lambda_{2}^{* *}+\mu, A_{2}=\lambda_{1}^{* *} \theta_{1}+\mu$ and $A_{3}=\lambda_{2}^{* *} \theta_{2}+\mu$.

The equilibria of the model (1) can then be obtained by finding the fixed-points of the equation

$$
x=\Phi(x)=\left(\begin{array}{c}
\phi_{1}\left(\lambda_{1}^{* *}, \lambda_{2}^{* *}\right) \\
\phi_{2}\left(\lambda_{1}^{* *}, \lambda_{2}^{* *}\right)
\end{array}\right) \text {, where } x=\left(\begin{array}{c}
\lambda_{1}^{* *} \\
\\
\lambda_{2}^{* *}
\end{array}\right) .
$$




\subsection{Strain 1-only Boundary Equilibrium $\left(\mathcal{E}_{1}\right)$}

Setting $\lambda_{2}^{* *}=0$ in (11) gives the following general form of the strain 1-only boundary equilibrium (denoted by $\mathcal{E}_{1}$ )

$$
\mathcal{E}_{1}=\left(S_{1}^{*}, E_{1}^{*}, I_{1}^{*}, R_{1}^{*}, 0,0,0,0,0,0,0,0\right)
$$

where,

$$
S_{1}^{*}=\frac{\Pi}{\lambda_{1}^{* *}+\mu}, \quad E_{1}^{*}=\frac{\lambda_{1}^{* *} \Pi}{K_{1}\left(\lambda_{1}^{* *}+\mu\right)}, \quad I_{1}^{*}=\frac{\lambda_{1}^{* *} \Pi \sigma_{1}}{K_{1} K_{2}\left(\lambda_{1}^{* *}+\mu\right)}, \quad R_{1}^{*}=\frac{\lambda_{1}^{* *} \Pi \sigma_{1} \gamma_{1}}{K_{1} K_{2} \mu\left(\lambda_{1}^{* *}+\mu\right)}
$$

We claim the following.

Theorem 4 The model (1) has a unique and LAS strain 1-only boundary equilibrium, $\mathcal{E}_{1}$, whenever $\mathcal{R}_{02}<1<\mathcal{R}_{01}$.

Proof. Consider, first of all, the model (1) with strain 2-only (i.e., the case where all components of Strain 1 are removed from the model). The following result can be proven (see Appendix B for the proof):

Lemma 3 In the absence of Strain 1, the DFE of the model (1) with strain 2-only is GAS in $\Omega$ whenever $\mathcal{R}_{02} \leq 1$.

It is clear from the fixed-point system, (12) with $(11)$, that $\phi_{2}\left(\lambda_{1}^{* *}, 0\right)=0$. Thus, a fixed-point of $\phi_{1}\left(\lambda_{1}^{* *}, \lambda_{2}^{* *}\right)$ is obtained by solving the equation $\phi_{1}\left(\lambda_{1}^{* *}, 0\right)=\lambda_{1}^{* *}$. It follows, after some algebraic manipulations, that $\lambda_{1}^{* *}$ is the root of the equation

$$
x_{1} \lambda_{1}^{* *}+y_{1}=0,
$$

where, $x_{1}=\sigma_{1}\left(\mu+\gamma_{1}\right)+\mu K_{2}$ and $y_{1}=\mu K_{1} K_{2}\left(1-\mathcal{R}_{01}\right)$. Clearly, the coefficient $x_{1}$ is always positive, and $y_{1}$ is positive (negative) if $\mathcal{R}_{01}$ is less than (greater than) unity, respectively. Further, it should be stated that for $\mathcal{E}_{1}$ to exist, it is necessary that strain 2 does not (i.e., $\mathcal{R}_{02}<1$ ). Thus, a unique strain 1 -only boundary equilibrium exists whenever $\mathcal{R}_{02}<1<\mathcal{R}_{01}$.

Let $\mathcal{R}_{02}<1$ (so that strain 2 dies out, in line with Lemma 3). The local stability property of $\mathcal{E}_{1}$ is established by evaluating Jacobian of the system (11) at $\left(\lambda_{1}^{* *}, \lambda_{2}^{* *}\right)$. This gives.

$J\left(\lambda_{1}^{* *}, \lambda_{2}^{* *}\right)=\left[\begin{array}{cc}\frac{\partial \phi_{1}\left(\lambda_{1}^{* *}, \lambda_{2}^{* *}\right)}{\partial \lambda_{1}^{* *}} & \frac{\partial \phi_{1}\left(\lambda_{1}^{* *}, \lambda_{2}^{* *}\right)}{\partial \lambda_{2}^{* *}} \\ \frac{\partial \phi_{2}\left(\lambda_{1}^{* *}, \lambda_{2}^{* *}\right)}{\partial \lambda_{1}^{* *}} & \frac{\partial \phi_{2}\left(\lambda_{1}^{* *}, \lambda_{2}^{* *}\right)}{\partial \lambda_{2}^{* *}}\end{array}\right]$ 
so that,

$$
J\left(\lambda_{1}^{* *}, 0\right)=\left[\begin{array}{cc}
\frac{1}{\mathcal{R}_{01}} & \left.\frac{\partial \phi_{1}\left(\lambda_{1}^{* *}, \lambda_{2}^{* *}\right)}{\partial \lambda_{2}^{* *}}\right|_{\left(\lambda_{1}^{* *}, 0\right)} \\
0 & \frac{\mathcal{R}_{02}}{\mathcal{R}_{01}}
\end{array}\right] .
$$

For stability, we require $\frac{1}{\mathcal{R}_{01}}<1$ (i.e., $\mathcal{R}_{01}>1$ ) and $\frac{\mathcal{R}_{02}}{\mathcal{R}_{01}}<1$ (i.e., $\mathcal{R}_{02}<\mathcal{R}_{01}$ ). Combining all these, and noting that $\mathcal{R}_{02} \leq 1$, shows that the boundary equilibrium, $\mathcal{E}_{1}$, is LAS provided $\mathcal{R}_{02}<1<\mathcal{R}_{01}$.

The boundary equilibrium $\mathcal{E}_{1}$ can be shown to be GAS for a special case as below. Consider the model (1) with the associated disease-induced mortality rates $\left(\delta_{1}, \delta_{2}, \delta_{12}, \delta_{21}\right)$ set to zero. Setting $\delta_{1}=\delta_{2}=\delta_{12}=\delta_{21}=0$ in (1) gives $N(t) \rightarrow \frac{\Pi}{\mu}$ as $t \rightarrow \infty$. Let $\tilde{\beta}_{i}=\frac{\mu \beta_{i}}{\Pi}$ and $\tilde{\beta}_{i j}=\frac{\mu \beta_{i j}}{\Pi}$, so that

$\lambda_{1}=\tilde{\beta}_{1}\left(\eta_{1} E_{1}+I_{1}\right)+\tilde{\beta}_{21}\left(\eta_{21} E_{21}+I_{21}\right)$ and $\lambda_{2}=\tilde{\beta}_{2}\left(\eta_{2} E_{2}+I_{2}\right)+\tilde{\beta}_{12}\left(\eta_{12} E_{12}+I_{12}\right)$.

Furthermore, let

$$
\begin{aligned}
\Omega_{0}= & \left\{\left(S, E_{1}, I_{1}, R_{1}, E_{2}, I_{2}, R_{2}, E_{12}, I_{12}, E_{21}, I_{21}, M\right) \in \Omega:\right. \\
& \left.E_{1}=I_{1}=E_{2}=I_{2}=E_{12}=I_{12}=E_{21}=I_{21}=0\right\} .
\end{aligned}
$$

We claim the following result.

Theorem 5 Let $\mathcal{R}_{02}<1$, so that $\lambda_{2}^{* *}=0$ and strain 2 dies out. The unique strain 1-only boundary equilibrium, $\mathcal{E}_{1}$ of the model (1) with (14), is GAS in $\Omega \backslash \Omega_{0}$, whenever $\mathcal{R}_{02}<1<\mathcal{R}_{01}$.

Proof. Consider the model (1) with (14). Further, let $\mathcal{R}_{02}<1<\mathcal{R}_{01}$, so that the boundary equilibrium $\mathcal{E}_{1}$ exists (Theorem 4 ) and strain-2 dies out (Lemma 3 ). Consider the following non-linear Lyapunov function, of Goh-Volterra type (functions of this type have been used in the mathematical ecology/epidemiology literature, such as those in $[7,22,26,28])$ :

$$
\begin{aligned}
\mathcal{F} & =S-S^{* *}-S^{* *} \ln \left(\frac{S}{S^{* *}}\right)+E_{1}-E_{1}^{* *}-E_{1}^{* *} \ln \left(\frac{E_{1}}{E_{1}^{* *}}\right) \\
& +\left(\frac{K_{1}-\tilde{\beta}_{1} \eta_{1} S^{* *}}{\sigma_{1}}\right)\left[I_{1}-I_{1}^{* *}-I_{1}^{* *} \ln \left(\frac{I_{1}}{I_{1}^{* *}}\right)\right],
\end{aligned}
$$

with Lyapunov derivative,

$$
\dot{\mathcal{F}}=\dot{S}-\frac{S^{* *}}{S} \dot{S}+\dot{E}_{1}-\frac{E_{1}^{* *}}{E_{1}} \dot{E}_{1}+\left(\frac{K_{1}-\tilde{\beta}_{1} \eta_{1} S^{* *}}{\sigma_{1}}\right)\left(\dot{I}_{1}-\frac{I_{1}^{* *}}{I_{1}} \dot{I}_{1}\right),
$$


so that,

$$
\begin{aligned}
\dot{\mathcal{F}} & =\Pi-\lambda_{1} S-\mu S-\frac{S^{* *}}{S}\left(\Pi-\lambda_{1} S-\mu S\right)+\lambda_{1} S-K_{1} E_{1}-\frac{E_{1}^{* *}}{E_{1}}\left(\lambda_{1} S-K_{1} E_{1}\right) \\
& +\left(\frac{K_{1}-\tilde{\beta}_{1} \eta_{1} S^{* *}}{\sigma_{1}}\right)\left[\sigma_{1} E_{1}-K_{2} I_{1}-\frac{I_{1}^{* *}}{I_{1}}\left(\sigma_{1} E_{1}-K_{2} I_{1}\right)\right] .
\end{aligned}
$$

Hence,

$$
\begin{aligned}
\dot{\mathcal{F}} & =\Pi\left(1-\frac{S^{* *}}{S}\right)+\mu S^{* *}\left(1-\frac{S}{S^{* *}}\right)+\tilde{\beta}_{1} S^{* *} I_{1}+\tilde{\beta}_{2} S^{* *} I_{2} \\
& -\tilde{\beta}_{1} \eta_{1} S E_{1}^{* *}-\tilde{\beta}_{1} S I_{1} \frac{E_{1}^{* *}}{E_{1}}+K_{1} E_{1}^{* *}-\frac{K_{1} K_{2} I_{1}}{\sigma_{1}}+\frac{\tilde{\beta}_{1} \eta_{1} S^{* *} K_{2} I_{1}}{\sigma_{1}} \\
& -K_{1} E_{1} \frac{I_{1}^{* *}}{I_{1}}+\frac{K_{1} K_{2} I_{1}^{* *}}{\sigma_{1}}+\tilde{\beta}_{1} \eta_{1} S^{* *} E_{1} \frac{I_{1}^{* *}}{I_{1}}-\frac{\tilde{\beta}_{1} \eta_{1} S^{* *} K_{2} I_{1}^{* *}}{\sigma_{1}} .
\end{aligned}
$$

It can be shown from the model (1) with (14) and $\lambda_{2}^{* *}=0$, at endemic steady-state, that

$$
\begin{aligned}
& \Pi=\tilde{\beta}_{1}\left(\eta_{1} E_{1}^{* *}+I_{1}^{* *}\right) S^{* *}+\mu S^{* *} \\
& K_{1} E_{1}^{* *}=\tilde{\beta}_{1}\left(\eta_{1} E_{1}^{* *}+I_{1}^{* *}\right) S^{* *}, \sigma_{1} E_{1}^{* *}=K_{2} I_{1}^{* *}, \gamma_{1} I_{1}^{* *}=\mu R_{1}^{* *}
\end{aligned}
$$

Substituting the relations in (16) into (15) gives

$$
\begin{aligned}
\dot{\mathcal{F}} & =\left[\tilde{\beta}_{1} \eta_{1} S^{* *} E_{1}^{* *}+\tilde{\beta}_{1} S^{* *} I_{1}^{* *}+\mu S^{* *}\right]\left(1-\frac{S^{* *}}{S}\right)+\mu S^{* *}\left(1-\frac{S}{S^{* *}}\right) \\
& -\tilde{\beta}_{1} \eta_{1} S E_{1}^{* *}-\tilde{\beta}_{1} S I_{1} \frac{E_{1}^{* *}}{E_{1}} \tilde{\beta}_{1} \eta_{1} S^{* *} E_{1}^{* *}+\tilde{\beta}_{1} S^{* *} I_{1}^{* *}-\tilde{\beta}_{1} \eta_{1} S^{* *} E_{1}^{* *} \frac{I_{1}}{I_{1}^{* *}} \\
& -\tilde{\beta}_{1} S^{* *} I_{1}^{* *} \frac{E_{1} I_{1}^{* *}}{E_{1}^{* *} I_{1}}+\tilde{\beta}_{1} S^{* *} I_{1}^{* *},
\end{aligned}
$$

which can be simplified to,

$$
\begin{aligned}
& =\mu S^{* *}\left(2-\frac{S^{* *}}{S}-\frac{S}{S^{* *}}\right)+\tilde{\beta}_{1} \eta_{1} S^{* *} E_{1}^{* *}\left(2-\frac{S^{* *}}{S}-\frac{S}{S^{* *}}\right) \\
& +\tilde{\beta}_{1} S^{* *} I_{1}^{* *}\left(3-\frac{S^{* *}}{S}-\frac{E_{1} I_{1}^{* *}}{E_{1}^{* *} I_{1}}-\frac{I_{1} E_{1}^{* *} S}{I_{1}^{* *} E_{1} S^{* *}}\right) .
\end{aligned}
$$

Finally, since the arithmetic mean exceeds the geometric mean, the following inequalities from (17) hold:

$$
2-\frac{S^{* *}}{S}-\frac{S}{S^{* *}} \leq 0, \quad 3-\frac{S^{* *}}{S}-\frac{E_{1} I_{1}^{* *}}{E_{1}^{* *} I_{1}}-\frac{I_{1} E_{1}^{* *} S}{I_{1}^{* *} E_{1} S^{* *}} \leq 0 .
$$


Thus, $\dot{\mathcal{F}} \leq 0$ for $\mathcal{R}_{01}>1$. Hence, $\mathcal{F}$ is a Lyapunov function on $\Omega$. The proof is completed as in the proof of Theorem 3 .

Figures 2A-B depict simulations for the case where $\mathcal{R}_{02}<1<\mathcal{R}_{01}$, showing the persistence of strain 1 , while strain 2 dies out.

\subsection{Strain 2-only Boundary Equilibrium $\left(\mathcal{E}_{2}\right)$}

Setting $\lambda_{1}^{* *}=0$ in (11) gives the following general form of the strain 2-only boundary equilibrium (denoted by $\mathcal{E}_{2}$ )

$$
\mathcal{E}_{2}=\left(S_{2}^{*}, 0,0,0, E_{2}^{*}, I_{2}^{*}, R_{2}^{*}, 0,0,0,0,0\right)
$$

where,

$$
S_{2}^{*}=\frac{\Pi}{\lambda_{2}^{* *}+\mu}, \quad E_{2}^{*}=\frac{\lambda_{2}^{* *} \Pi}{K_{3}\left(\lambda_{2}^{* *}+\mu\right)}, \quad I_{2}^{*}=\frac{\lambda_{2}^{* *} \Pi \sigma_{2}}{K_{3} K_{4}\left(\lambda_{2}^{* *}+\mu\right)}, \quad R_{2}^{*}=\frac{\lambda_{2}^{* *} \Pi \sigma_{2} \gamma_{2}}{K_{3} K_{4} \mu\left(\lambda_{2}^{* *}+\mu\right)}
$$

The following result can be shown using the approach in Section 3.1.

Theorem 6 The model (1) has a unique and LAS strain 2-only boundary equilibrium, $\mathcal{E}_{2}$, whenever $\mathcal{R}_{01}<1<\mathcal{R}_{02}$.

Furthermore, the result below holds (see Appendix C for the proof):

Theorem 7 The unique strain 2-only boundary equilibrium $\mathcal{E}_{2}$, of the model (1) with (14), is GAS in $\Omega \backslash \Omega_{0}$, whenever $\mathcal{R}_{01}<1<\mathcal{R}_{02}$.

Numerical simulations for the case when $\mathcal{R}_{01}<1<\mathcal{R}_{02}$, depicted in Figure 3, show that strain 1 dies out while strain 2 establishes itself at steady-state. Thus, the model undergoes competitive exclusion, with Strain $i$ driving out Strain $j$ if $\mathcal{R}_{0 i}>1>$ $\mathcal{R}_{0 j}(i, j=1,2 ; i \neq j)$. It should be mentioned that the results for the boundary equilibria of the model (1) are consistent with those reported in [19, 20, 23], associated with the modeling of the spread of dengue fever. The numerical simulations carried out in this study are based on using the parameter values tabulated in Table 2 , which are consistent with the transmission dynamics of influenza (in particular, $1.5<\mathcal{R}_{01}, \mathcal{R}_{02}<$ $5.4[15,16,17,18,21,26,35,36])$.

Exploring the possible existence of co-existence equilibria of the model (1) theoretically proved to be quite challenging (due to its large size and nonlinearity). However, numerical simulations suggest that the model (1) has a co-existence (endemic) equilibrium (where both strains co-exist) whenever $\mathcal{R}_{0 i}>\mathcal{R}_{0 j}>1 ; i, j=1,2 ; i \neq j$ ). 
Furthermore, although the strain with the higher reproduction number dominates the other, the two strains always co-exist (i.e., the strain with the higher reproduction number does not drive out the other strain to extinction) as depicted in Figures $4 \mathrm{~A}$ and B. The two strains co-exist for the case when $\mathcal{R}_{0 i}=\mathcal{R}_{0 j}>1(i \neq j)$ (Figure 4C). This suggest the following conjecture.

Conjecture 1 The model (1) has a unique co-existence equilibrium whenever $\mathcal{R}_{0 i}>$ $\mathcal{R}_{0 j}>1$.

It is worth mentioning, unlike in Theorems 5 and 7 , that the global-asymptotic stability of the associated boundary equilibria of the model considered in [29] was not established.

\subsection{Existence and Stability of Co-existence (Endemic) Equi- libria: Special Case $\theta_{1}=\theta_{2}=0$}

The existence of co-existence (endemic) equilibrium of the model (1) is explored for the special case where infection with one strain confers complete immunity against infection with the other strain (i.e. $\theta_{1}=\theta_{2}=0$ ). Consider the model (1) with $\theta_{1}=\theta_{2}=0$ (or, equivalently, the reduced model $(7)$ ). Let $\mathcal{E}_{2}=\left(S^{* *}, E_{1}^{* *}, I_{1}^{* *}, R_{1}^{* *}, E_{2}^{* *}, I_{2}^{* *}, R_{2}^{* *}\right)$ represent any arbitrary equilibrium of the reduced model (7). It should be recalled that, in this setting,

$$
\lambda_{1}^{* *}=\frac{\beta_{1}\left(\eta_{2} E_{1}^{* *}+I_{1}^{* *}\right)}{N^{* *}} \text { and } \lambda_{2}^{* *}=\frac{\beta_{2}\left(\eta_{2} E_{2}^{* *}+I_{2}^{* *}\right)}{N^{* *}} .
$$

Substituting the expressions in (11) into (18) gives,

$$
\lambda_{1}^{* *}=\frac{\mu K_{3} K_{4} \lambda_{1}^{* *} \beta_{1}\left(\eta_{1} K_{2}+\sigma_{1}\right)}{\mu K_{1} K_{2} K_{3} K_{4}+\lambda_{1}^{* *} Q_{1}+\lambda_{2}^{* *} Q_{2}} \text { and } \lambda_{2}^{* *}=\frac{\mu K_{1} K_{2} \lambda_{2}^{* *} \beta_{2}\left(\eta_{2} K_{4}+\sigma_{2}\right)}{\mu K_{1} K_{2} K_{3} K_{4}+\lambda_{1}^{* *} Q_{1}+\lambda_{2}^{* *} Q_{2}},
$$

where, $Q_{1}=K_{3} K_{4}\left[\sigma_{1}\left(\mu+\gamma_{1}\right)+K_{2} \mu\right]$ and $Q_{2}=K_{1} K_{2}\left[\sigma_{2}\left(\mu+\gamma_{2}\right)+K_{4} \mu\right]$.

The expressions in (19) can be re-written as:

$$
\begin{aligned}
& \lambda_{1}^{* *}=\frac{\mu K_{3} K_{4} \lambda_{1}^{* *} \beta_{1}\left(\eta_{1} K_{2}+\sigma_{1}\right)}{\mu K_{1} K_{2} K_{3} K_{4}+\lambda_{1}^{* *} Q_{1}+\lambda_{2}^{* *} Q_{2}} \equiv \frac{\lambda_{1}^{* *} \mu K_{1} K_{2} K_{3} K_{4} \mathcal{R}_{01}}{\mu K_{1} K_{2} K_{3} K_{4}+\lambda_{1}^{* *} Q_{1}+\lambda_{2}^{* *} Q_{2}}, \\
& \lambda_{2}^{* *}=\frac{\mu K_{1} K_{2} \lambda_{2}^{* *} \beta_{2}\left(\eta_{2} K_{4}+\sigma_{2}\right)}{\mu K_{1} K_{2} K_{3} K_{4}+\lambda_{1}^{* *} Q_{1}+\lambda_{2}^{* *} Q_{2}} \equiv \frac{\lambda_{2}^{* *} \mu K_{1} K_{2} K_{3} K_{4} \mathcal{R}_{02}}{\mu K_{1} K_{2} K_{3} K_{4}+\lambda_{1}^{* *} Q_{1}+\lambda_{2}^{* *} Q_{2}} .
\end{aligned}
$$

It follows from (20) that

$$
\begin{aligned}
& \lambda_{1}^{* *} Q_{1}+\lambda_{2}^{* *} Q_{2}=\mu K_{1} K_{2} K_{3} K_{4}\left(\mathcal{R}_{01}-1\right), \\
& \lambda_{1}^{* *} Q_{1}+\lambda_{2}^{* *} Q_{2}=\mu K_{1} K_{2} K_{3} K_{4}\left(\mathcal{R}_{02}-1\right) .
\end{aligned}
$$


Since the left-hand sides of the equations in (21) are always positive, it is necessary that $\mathcal{R}_{01}>1$ and $\mathcal{R}_{02}>1$. If $\mathcal{R}_{01} \neq \mathcal{R}_{02}$, then the system (21) is inconsistent (and there is no positive co-existence equilibrium in this case). Hence, for the two equations in (21) to be consistent, it is necessary that $\mathcal{R}_{01}=\mathcal{R}_{02}>1$. It follows then that a continuum (family) of endemic equilibria will arise in this case. That is, setting $\mathcal{R}_{01}=\mathcal{R}_{02}=\mathcal{R}_{0 i}>1$ implies that

$$
\lambda_{1}^{* *} Q_{1}+\lambda_{2}^{* *} Q_{2}=\mu K_{1} K_{2} K_{3} K_{4}\left(\mathcal{R}_{0 i}-1\right)
$$

so that $0<\lambda_{1}^{* *}<\frac{\mu K_{1} K_{2} K_{3} K_{4}\left(\mathcal{R}_{01}-1\right)}{Q_{1}}$ and $0<\lambda_{2}^{* *}<\frac{\mu K_{1} K_{2} K_{3} K_{4}\left(\mathcal{R}_{02}-1\right)}{Q_{2}}$. This result is summarized below.

Theorem 8 The model (1) with $\theta_{1}=\theta_{2}=0$ (or, equivalently, (7)) has a continuum of positive co-existence equilibria, denoted by $E_{c}^{n}\left(n \in \mathbb{Z}_{+}\right)$, whenever the following conditions hold

(i) $\mathcal{R}_{01}=\mathcal{R}_{02}=\mathcal{R}_{0 i}>1$,

(ii) $0<\lambda_{1}^{* *}<\frac{\mu K_{1} K_{2} K_{3} K_{4}\left(\mathcal{R}_{01}-1\right)}{Q_{1}}$

(iii) $0<\lambda_{2}^{* *}<\frac{\mu K_{1} K_{2} K_{3} K_{4}\left(\mathcal{R}_{02}-1\right)}{Q_{2}}$,

and no co-existence equilibria otherwise.

We claim the following result.

Theorem 9 Let $\mathcal{R}_{c}^{n}=\frac{L_{0}+\sqrt{L_{0}^{2}-4 L_{1}}}{2}, \quad\left(n \in \mathbb{Z}_{+}\right)$, with

$$
L_{0}=\left.\left(\frac{\partial \phi_{1}}{\partial \lambda_{1}^{* *}}+\frac{\partial \phi_{2}}{\partial \lambda_{2}^{* *}}\right)\right|_{\left(\lambda_{1}^{* *}, \lambda_{2}^{* *}\right)} \text { and } L_{1}=\left.\left(\frac{\partial \phi_{1}}{\partial \lambda_{1}^{* *}} \frac{\partial \phi_{2}}{\partial \lambda_{2}^{* *}}-\frac{\partial \phi_{1}}{\partial \lambda_{2}^{* *}} \frac{\partial \phi_{2}}{\partial \lambda_{1}^{* *}}\right)\right|_{\left(\lambda_{1}^{* *}, \lambda_{2}^{* *}\right)} .
$$

Then, the family of co-existence equilibria, $E_{c}^{n}$, of (7) is LAS whenever $\mathcal{R}_{c}^{n}<1$, for each $n \in \mathbb{Z}_{+}$.

Proof. Evaluating the Jacobian of $\Phi$ at each $\left(\lambda_{1}^{* *}, \lambda_{2}^{* *}\right)$ in the regions (i) to (iii), gives $J\left(\lambda_{1}^{* *}, \lambda_{2}^{* *}\right)=\left[\begin{array}{cc}\frac{\partial \phi_{1}\left(\lambda_{1}^{* *}, \lambda_{2}^{* *}\right)}{\partial \lambda_{1}^{* *}} & \frac{\partial \phi_{1}\left(\lambda_{1}^{* *}, \lambda_{2}^{* *}\right)}{\partial \lambda_{2}^{* *}} \\ \frac{\partial \phi_{2}\left(\lambda_{1}^{* *}, \lambda_{2}^{* *}\right)}{\partial \lambda_{1}^{* *}} & \frac{\partial \phi_{2}\left(\lambda_{1}^{* *}, \lambda_{2}^{* *}\right)}{\partial \lambda_{2}^{* *}},\end{array}\right]$

with eigenvalues given by the roots of the characteristic polynomials 


$$
\Lambda^{2}-\Lambda L_{0}+L_{1}=0
$$

It can be shown that the dominant eigenvalue of $J\left(\lambda_{1}^{* *}, \lambda_{2}^{* *}\right)$ is $\Lambda=\left|\mathcal{R}_{c}^{n}\right|$. Thus, the family of co-existence endemic equilibria, $E_{c}^{n}$, is LAS whenever $\left|\mathcal{R}_{c}^{n}\right|<1$ for each $n$.

Figures $5 \mathrm{~A}$ and $\mathrm{B}$ illustrate the existence of the continuum of co-existence equilibria when the two reproduction numbers are equal and greater than unity $\left(\mathcal{R}_{01}=\mathcal{R}_{02}>\right.$ 1). It is worth stating that the phenomenon of having infinitely many co-existence equilibria has been observed in other epidemiological settings, such as in the study of the dynamics of multiple strains of TB [11], dengue fever [23] and HIV [39].

\subsubsection{Global stability of continuum of co-existence equilibria: special case}

$$
\delta_{1}=\delta_{2}=0
$$

Here, the global stability of the continuum of co-existence equilibria of the model (7) is given for the special case where the disease-induced mortality rates $\left(\delta_{1}\right.$ and $\left.\delta_{2}\right)$ are set to zero. Setting $\delta_{1}=\delta_{2}=0$ in (7) shows that $N \rightarrow \frac{\Pi}{\mu}$ as $t \rightarrow \infty$. Furthermore, let $\tilde{\beta}_{1}=\frac{\beta_{1} \mu}{\Pi}$ and $\tilde{\beta}_{2}=\frac{\beta_{2} \mu}{\Pi}$. It follows from (7), with $N=\Pi / \mu$, that

$$
\lambda_{1}=\tilde{\beta}_{1}\left(\eta_{1} E_{1}+I_{1}\right) \text { and } \lambda_{2}=\tilde{\beta}_{2}\left(\eta_{2} E_{2}+I_{2}\right) .
$$

Define

$$
\Gamma_{0}=\left\{\left(S, E_{1}, I_{1}, R_{1}, E_{2}, I_{2}, R_{2}\right) \in \Gamma: E_{1}=I_{1}=E_{2}=I_{2}=0\right\}
$$

the stable manifold of the $\operatorname{DFE}\left(\mathcal{E}_{0}^{r}\right)$. We claim the following.

Theorem 10 Consider the model (7) with (23). The continuum of co-existence equilibria of the model is $G A S$ in $\Gamma \backslash \Gamma_{0}$ if $\mathcal{R}_{01}=\mathcal{R}_{02}>1$.

Proof. Consider the model (7) with (23) and $\mathcal{R}_{01}=\mathcal{R}_{02}=\mathcal{R}_{0}>1$, so that the continuum of equilibria of the model exists. Further, consider the following Lyapunov function:

$$
\begin{aligned}
\mathcal{F} & =S-S^{* *}-S^{* *} \ln \left(\frac{S}{S^{* *}}\right)+E_{1}-E_{1}^{* *}-E_{1}^{* *} \ln \left(\frac{E_{1}}{E_{1}^{* *}}\right)+E_{2}-E_{2}^{* *}-E_{2}^{* *} \ln \left(\frac{E_{2}}{E_{2}^{* *}}\right) \\
& +\left(\frac{K_{1}-\tilde{\beta}_{1} \eta_{1} S^{* *}}{\sigma_{1}}\right)\left[I_{1}-I_{1}^{* *}-I_{1}^{* *} \ln \left(\frac{I_{1}}{I_{1}^{* *}}\right)\right]+\left(\frac{K_{3}-\tilde{\beta}_{2} \eta_{2} S^{* *}}{\sigma_{2}}\right)\left[I_{2}-I_{2}^{* *}-I_{2}^{* *} \ln \left(\frac{I_{2}}{I_{2}^{* *}}\right)\right]
\end{aligned}
$$


with Lyapunov derivative,

$$
\begin{aligned}
\dot{\mathcal{F}} & =\dot{S}-\frac{S^{* *}}{S} \dot{S}+\dot{E}_{1}-\frac{E_{1}^{* *}}{E_{1}} \dot{E}_{1}+\dot{E}_{2}-\frac{E_{2}^{* *}}{E_{2}} \dot{E}_{2}+\left(\frac{K_{1}-\tilde{\beta}_{1} \eta_{1} S^{* *}}{\sigma_{1}}\right)\left(\dot{I}_{1}-\frac{I_{1}^{* *}}{I_{1}} \dot{I}_{1}\right) \\
& +\left(\frac{K_{3}-\tilde{\beta}_{2} \eta_{2} S^{* *}}{\sigma_{2}}\right)\left(\dot{I}_{2}-\frac{I_{2}^{* *}}{I_{2}} \dot{I}_{2}\right)
\end{aligned}
$$

so that,

$$
\begin{aligned}
\dot{\mathcal{F}} & =\Pi-\lambda_{1} S+\lambda_{2} S-\mu S-\frac{S^{* *}}{S}\left(\Pi-\lambda_{1} S+\lambda_{2} S-\mu S\right) \\
& +\lambda_{1} S-K_{1} E_{1}-\frac{E_{1}^{* *}}{E_{1}}\left(\lambda_{1} S-K_{1} E_{1}\right)+\lambda_{2} S-K_{3} E_{2}-\frac{E_{2}^{* *}}{E_{2}}\left(\lambda_{2} S-K_{3} E_{2}\right) \\
& +\left(\frac{K_{1}-\tilde{\beta}_{1} \eta_{1} S^{* *}}{\sigma_{1}}\right)\left[\sigma_{1} E_{1}-K_{2} I_{1}-\frac{I_{1}^{* *}}{I_{1}}\left(\sigma_{1} E_{1}-K_{2} I_{1}\right)\right] \\
& +\left(\frac{K_{3}-\tilde{\beta}_{2} \eta_{2} S^{* *}}{\sigma_{2}}\right)\left[\sigma_{2} E_{2}-K_{4} I_{2}-\frac{I_{2}^{* *}}{I_{2}}\left(\sigma_{2} E_{2}-K_{4} I_{2}\right)\right] .
\end{aligned}
$$

Hence,

$$
\begin{aligned}
\dot{\mathcal{F}} & =\Pi\left(1-\frac{S^{* *}}{S}\right)+\mu S^{* *}\left(1-\frac{S}{S^{* *}}\right)+\tilde{\beta}_{1} S^{* *} I_{1}+\tilde{\beta}_{2} S^{* *} I_{2} \\
& -\tilde{\beta}_{1} \eta_{1} S E_{1}^{* *}-\tilde{\beta}_{1} S I_{1} \frac{E_{1}^{* *}}{E_{1}}+K_{1} E_{1}^{* *}-\tilde{\beta}_{2} \eta_{2} S E_{2}^{* *}-\tilde{\beta}_{2} S I_{2} \frac{E_{2}^{* *}}{E_{2}}+K_{3} E_{2}^{* *} \\
& -\frac{K_{1} K_{2} I_{1}}{\sigma_{1}}+\frac{\tilde{\beta}_{1} \eta_{1} S^{* *} K_{2} I_{1}}{\sigma_{1}}-K_{1} E_{1} \frac{I_{1}^{* *}}{I_{1}}+\frac{K_{1} K_{2} I_{1}^{* *}}{\sigma_{1}}+\tilde{\beta}_{1} \eta_{1} S^{* *} E_{1} \frac{I_{1}^{* *}}{I_{1}}-\frac{\tilde{\beta}_{1} \eta_{1} S^{* *} K_{2} I_{1}^{* *}}{\sigma_{1}} \\
& -\frac{K_{3} K_{4} I_{2}}{\sigma_{2}}+\frac{\tilde{\beta}_{2} \eta_{2} S^{* *} K_{4} I_{2}}{\sigma_{2}}-K_{3} E_{2} \frac{I_{2}^{* *}}{I_{2}}+\frac{K_{3} K_{4} I_{2}^{* *}}{\sigma_{2}}+\tilde{\beta}_{2} \eta_{2} S^{* *} E_{2} \frac{I_{2}^{* *}}{I_{2}}-\frac{\tilde{\beta}_{2} \eta_{2} S^{* *} K_{4} I_{2}^{* *}}{\sigma_{2}} .
\end{aligned}
$$

It can be shown from the model (7) with (23), at endemic steady-state, that

$$
\begin{aligned}
& \Pi=\tilde{\beta}_{1}\left(\eta_{1} E_{1}^{* *}+I_{1}^{* *}\right) S^{* *}+\tilde{\beta}_{2}\left(\eta_{2} E_{2}^{* *}+I_{2}^{* *}\right) S^{* *}+\mu S^{* *}, \\
& K_{1} E_{1}^{* *}=\tilde{\beta}_{1}\left(\eta_{1} E_{1}^{* *}+I_{1}^{* *}\right) S^{* *}, \sigma_{1} E_{1}^{* *}=K_{2} I_{1}^{* *}, \gamma_{1} I_{1}^{* *}=\mu R_{1}{ }^{* *} \\
& K_{3} E_{2}^{* *}=\tilde{\beta}_{2}\left(\eta_{2} E_{2}^{* *}+I_{2}^{* *}\right) S^{* *}, \sigma_{2} E_{2}^{* *}=K_{4} I_{2}^{* *}, \gamma_{2} I_{2}^{* *}=\mu R_{2}{ }^{* *} .
\end{aligned}
$$

Substituting the relations in (25) into (24) gives

$$
\begin{aligned}
\dot{\mathcal{F}} & =\left[\tilde{\beta}_{1} \eta_{1} S^{* *} E_{1}^{* *}+\tilde{\beta}_{1} S^{* *} I_{1}^{* *}+\tilde{\beta}_{2} S^{* *} \eta_{2} E_{2}^{* *}+\tilde{\beta}_{2} S^{* *} I_{2}^{* *}+\mu S^{* *}\right]\left(1-\frac{S}{S}\right)+ \\
& \mu S^{* *}\left(1-\frac{S}{S^{* *}}\right)-\tilde{\beta}_{1} \eta_{1} S E_{1}^{* *}-\tilde{\beta}_{1} S I_{1} \frac{E_{1}^{* *}}{E_{1}} \tilde{\beta}_{1} \eta_{1} S^{* *} E_{1}^{* *}+\tilde{\beta}_{1} S^{* *} I_{1}^{* *}-\tilde{\beta}_{2} \eta_{2} S E_{2}^{* *}- \\
& \tilde{\beta}_{2} S I_{2} \frac{E_{2}^{* *}}{E_{2}}+\tilde{\beta}_{2} S^{* *} \eta_{2} E_{2}^{* *}+\tilde{\beta}_{2} S^{* *} I_{2}^{* *}-\tilde{\beta}_{1} \eta_{1} S^{* *} E_{1}^{* *} \frac{I_{1}}{I_{1}^{* *}}-\tilde{\beta}_{1} S^{* *} I_{1}^{* *} \frac{E_{1} I_{1}^{* *}}{E_{1}^{* *} I_{1}}+ \\
& \tilde{\beta}_{1} S^{* *} I_{1}^{* *}-\tilde{\beta}_{2} S^{* *} I_{2}^{* *} \frac{E_{2} I_{2}^{* *}}{E_{2}^{* *} I_{2}}+\tilde{\beta}_{2} S^{* *} I_{2}^{* *}
\end{aligned}
$$


which can be simplified to,

$$
\begin{aligned}
& =\mu S^{* *}\left(2-\frac{S^{* *}}{S}-\frac{S}{S^{* *}}\right)+\tilde{\beta}_{1} \eta_{1} S^{* *} E_{1}^{* *}\left(2-\frac{S^{* *}}{S}-\frac{S}{S^{* *}}\right)+\tilde{\beta}_{2} \eta_{2} S^{* *} E_{2}^{* *}\left(2-\frac{S^{* *}}{S}-\frac{S}{S^{* *}}\right) \\
& +\tilde{\beta}_{1} S^{* *} I_{1}^{* *}\left(3-\frac{S^{* *}}{S}-\frac{E_{1} I_{1}^{* *}}{E_{1}^{* *} I_{1}}-\frac{I_{1} E_{1}^{* *} S}{I_{1}^{* *} E_{1} S^{* *}}\right)+\tilde{\beta}_{2} S^{* *} I_{2}^{* *}\left(3-\frac{S^{* *}}{S}-\frac{E_{2} I_{2}^{* *}}{E_{2}^{* *} I_{2}}-\frac{I_{2} E_{2}^{* *} S}{I_{2}^{* *} E_{2} S^{* *}}\right) .
\end{aligned}
$$

Finally, since the arithmetic mean exceeds the geometric mean, the following inequalities from (27) hold:

$$
\begin{aligned}
& 2-\frac{S^{* *}}{S}-\frac{S}{S^{* *}} \leq 0,3-\frac{S^{* *}}{S}-\frac{E_{1} I_{1}^{* *}}{E_{1}^{* *} I_{1}}-\frac{I_{1} E_{1}^{* *} S}{I_{1}^{* *} E_{1} S^{* *}} \leq 0 \\
& 3-\frac{S^{* *}}{S}-\frac{E_{2} I_{2}^{* *}}{E_{2}^{* *} I_{2}}-\frac{I_{2} E_{2}^{* *} S}{I_{2}^{* *} E_{2} S^{* *}} \leq 0 .
\end{aligned}
$$

Furthermore, since $I_{1}^{* *}$ and $I_{2}^{* *}$ approaches their endemic state as $t \rightarrow \infty$, it follows that, $R_{1}(t) \rightarrow \frac{\gamma_{1} I_{1}^{* *}}{\mu}$ and $R_{2}(t) \rightarrow \frac{\gamma_{2} I_{2}^{* *}}{\mu}$. Thus, $\dot{\mathcal{F}} \leq 0$ for $\mathcal{R}_{01}=\mathcal{R}_{02}=\mathcal{R}_{0}>1$. Hence, $\mathcal{F}$ is a Lyapunov function on $\Gamma$. The proof is completed as in the proof of Theorem 3.

It should be emphasized that the global asymptotic stability results in Section 3 were not shown in some of the earlier related studies such as those in [23, 29].

\section{Conclusions}

A new deterministic model for the transmission dynamics of two strains of influenza is designed and rigorously analyzed. Some of the theoretical and epidemiological findings of the study are:

(i) The model (1) has a locally-asymptotically stable disease-free equilibrium whenever the associated reproduction number $\left(\mathcal{R}_{0}\right)$ is less than unity. This model undergoes the phenomenon of backward bifurcation, where the stable diseasefree equilibrium co-exists with a stable endemic equilibrium. This phenomenon is caused by the incomplete cross-immunity property of the model. For the case when infection with one strain confers complete immunity against the other, the DFE of the model is shown to be globally-asymptotically stable when $\mathcal{R}_{0}<1$.

(ii) For the case when infection with one strain confers incomplete immunity against the other, the model (1) exhibits the phenomenon of competitive exclusion, where strain $i$ drives out strain $j$ whenever the associated reproduction number $\mathcal{R}_{0 i}>$ $1>\mathcal{R}_{0 j}$, where $(i, j=1,2 ; i \neq j)$. Global asymptotic stability properties of 
the two boundary equilibria are established for the case where disease-induced mortality is zero.

(iii) Numerical simulations of the model (with partial cross-immunity) show that the two strains coexist with strain $i$ dominating, but not driving out strain $j$, if $\mathcal{R}_{0 i}>\mathcal{R}_{0 j}>1$

(iv) The model can have a continuum of co-existence equilibria when $\mathcal{R}_{0}>1$ for the case when infection with one strain confers complete immunity against the other strain. The continuum of equilibria is shown to be globally-asymptotically stable for a special case.

\section{Acknowledgments}

One of the authors (ABG) acknowledges, with thanks, the support in part of the Natural Science and Engineering Research Council (NSERC) and Mathematics of Information Technology and Complex Systems (MITACS) of Canada. SMG acknowledges the support, in part, of Research and Development Program (RDP), University of Pretoria. The authors are grateful to the anonymous Reviewers and the Handling Editor for their constructive comments. 


\section{Appendix A: Proof of Theorem 2}

Proof. Suppose

$$
\mathcal{E}_{e}=\left(S^{* *}, E_{1}^{* *}, I_{1}^{* *}, R_{1}^{* *}, E_{2}^{* *}, I_{2}^{* *}, R_{2}^{* *}, E_{12}^{* *}, I_{12}^{* *}, E_{21}^{* *}, I_{21}^{* *}, M^{* *}\right)
$$

represents any arbitrary endemic equilibrium of the model (1) (that is, an equilibrium in which at least one of the infected components is non-zero). The existence of backward bifurcation will be explored using the Centre Manifold theory [10, 13, 41]. To apply this theory, it is convenient to carry out the following change of variables. Let $S=$ $x_{1}, E_{1}=x_{2}, I_{1}=x_{3}, R_{1}=x_{4}, E_{2}=x_{5}, I_{2}=x_{6}, R_{2}=x_{7}, E_{12}=x_{8}, I_{12}=$ $x_{9}, E_{21}=x_{10}, I_{21}=x_{11}$, and $M=x_{12}$, so that $N=x_{1}+x_{2}+x_{3}+x_{4}+x_{5}+$ $x_{6}+x_{7}+x_{8}+x_{9}+x_{10}+x_{11}+x_{12}$. Further, by using the vector notation $X=$ $\left(x_{1}, x_{2}, x_{3}, x_{4}, x_{5}, x_{6}, x_{7}, x_{8}, x_{9}, x_{10}, x_{11}, x_{12}\right)^{T}$, the model (1) can be written in the form $\frac{d X}{d t}=F(X)$, with $\left(f_{1}, f_{2}, f_{3}, f_{4}, f_{5}, f_{6}, f_{7}, f_{8}, f_{9}, f_{10}, f_{11}, f_{12}\right)^{T}$, as follows:

$$
\begin{aligned}
\frac{d x_{1}}{d t} & \equiv f_{1}=\Pi+\xi x_{12}-\lambda_{1} x_{1}-\lambda_{2} x_{1}-\mu x_{1}, \\
\frac{d x_{2}}{d t} & \equiv f_{2}=\lambda_{1} x_{1}-K_{1} x_{2}, \\
\frac{d x_{3}}{d t} & \equiv f_{3}=\sigma_{1} x_{2}-K_{2} x_{3}, \\
\frac{d x_{4}}{d t} & \equiv f_{4}=\gamma_{1} x_{3}-\theta_{2} \lambda_{2} x_{4}-\mu x_{4}, \\
\frac{d x_{5}}{d t} & \equiv f_{5}=\lambda_{2} x_{1}-K_{3} x_{5}, \\
\frac{d x_{6}}{d t} & \equiv f_{6}=\sigma_{2} x_{5}-K_{4} x_{6}, \\
\frac{d x_{7}}{d t} & \equiv f_{7}=\gamma_{2} x_{6}-\theta_{1} \lambda_{1} x_{7}-\mu x_{7}, \\
\frac{d x_{8}}{d t} & \equiv f_{8}=\theta_{2} \lambda_{2} x_{4}-K_{5} x_{8}, \\
\frac{d x_{9}}{d t} & \equiv f_{9}=\sigma_{12} x_{8}-K_{6} x_{9}, \\
\frac{d x_{10}}{d t} & \equiv f_{10}=\theta_{1} \lambda_{1} x_{7}-K_{7} x_{10}, \\
\frac{d x_{11}}{d t} & \equiv f_{11}=\sigma_{21} x_{11}-K_{8} x_{11}, \\
\frac{d x_{12}}{d t} & \equiv f_{12}=\gamma_{12} x_{9}+\gamma_{21} x_{11}-K_{9} x_{12},
\end{aligned}
$$

with the forces of infection given by

$$
\lambda_{1}=\frac{\beta_{1}\left(\eta_{1} x_{2}+x_{3}\right)+\beta_{21}\left(\eta_{21} x_{10}+x_{11}\right)}{\sum_{i=1}^{12} x_{i}}, \lambda_{2}=\frac{\beta_{2}\left(\eta_{2} x_{5}+x_{6}\right)+\beta_{12}\left(\eta_{12} x_{8}+x_{9}\right)}{\sum_{i=1}^{12} x_{i}} .
$$


Consider the case when $\mathcal{R}_{0}=1$. Suppose, further, that $\beta_{1}=\beta_{1}^{*}$ is chosen as a bifurcation parameter. Solving for $\beta_{1}=\beta_{1}^{*}$ from $\mathcal{R}_{0}=1$ in (5) gives

$$
\beta_{1}=\beta_{1}^{*}=\frac{K_{1} K_{2}}{\eta_{1} K_{2}+\sigma_{1}} .
$$

The Jacobian of the transformed system (1) at the DFE, $\mathcal{E}_{0}$ with $\beta_{1}=\beta_{1}^{*}$, is given by

$$
J^{*}=\left[\begin{array}{cccccccccccc}
-\mu & -\beta_{1}^{*} \eta_{1} & -\beta_{1}^{*} & 0 & -\beta_{2} \eta_{2} & -\beta_{2} & 0 & -\beta_{3} \eta_{3} & -\beta_{3} & \beta_{4} \eta_{4} & -\beta_{4} & \xi \\
0 & \beta_{1}^{*} \eta_{1}-K_{1} & \beta_{1}^{*} & 0 & 0 & 0 & 0 & 0 & 0 & \beta_{4} \eta_{4} & \beta_{2} & 0 \\
0 & \sigma_{1} & -K_{2} & 0 & 0 & 0 & 0 & 0 & 0 & 0 & 0 & 0 \\
0 & 0 & \gamma_{1} & -\mu & 0 & 0 & 0 & 0 & 0 & 0 & 0 & 0 \\
0 & 0 & 0 & 0 & \beta_{2} \eta_{2}-K_{3} & \beta_{2} & 0 & \beta_{3} \eta_{3} & \beta_{3} & 0 & 0 & 0 \\
0 & 0 & 0 & 0 & \sigma_{2} & -K_{4} & 0 & 0 & 0 & 0 & 0 & 0 \\
0 & 0 & 0 & 0 & 0 & \gamma_{2} & -\mu & 0 & 0 & 0 & 0 & 0 \\
0 & 0 & 0 & 0 & 0 & 0 & 0 & -K_{5} & 0 & 0 & 0 & 0 \\
0 & 0 & 0 & 0 & 0 & 0 & 0 & \sigma_{3} & -K_{6} & 0 & 0 & 0 \\
0 & 0 & 0 & 0 & 0 & 0 & 0 & 0 & 0 & K_{7} & 0 & 0 \\
0 & 0 & 0 & 0 & 0 & 0 & 0 & 0 & 0 & \sigma_{4} & -K_{8} & 0 \\
0 & 0 & 0 & 0 & 0 & 0 & 0 & 0 & 0 & \gamma_{3} & \gamma_{4} & -K_{9}
\end{array}\right],
$$

where $K_{9}=\mu+\xi$. The Jacobian $\left(J^{*}\right)$ of the linearized system has a simple zero eigenvalue (with all other eigenvalues having negative real part). Hence, the Centre Manifold Theory $[10,13,41]$ can be used to analyse the dynamics of the system (29). In particular, Theorem 4.5 in [13] will be used.

In order to apply Theorem 4.5 in [13], the following computation are necessary. The right eigenvector of $\left.J\left(\mathcal{E}_{0}\right)\right|_{\beta_{1}=\beta_{1}^{*}}$ is given by $\boldsymbol{w}=\left(\omega_{1}, \omega_{2}, . ., \omega_{12}\right)^{T}$,

where,

$$
\begin{aligned}
& \omega_{1}=-\left[\left(\eta_{1} K_{2}+\sigma_{1}\right) \frac{\beta_{1}^{*}}{\sigma_{1}}+\left(\eta_{2} K_{4}+\sigma_{2}\right) \frac{\beta_{2} \omega_{6}}{\sigma_{2}}\right] \frac{1}{\mu}, \\
& \omega_{2}=\frac{K_{2}}{\sigma_{1}}, \omega_{3}=1, \omega_{4}=\frac{\gamma_{1}}{\mu}, \omega_{5}=\frac{K_{4} \omega_{6}}{\sigma_{2}}, \omega_{6}=\omega_{6}, \omega_{7}=\frac{\gamma_{2} \omega_{6}}{\mu}, \\
& \omega_{8}=\omega_{9}=\omega_{10}=\omega_{11}=\omega_{12}=0 .
\end{aligned}
$$

Similarly, the components of the left eigenvector of $J^{*}$ (corresponding to the zero eigenvalue), denoted by $\mathbf{v}=\left[v_{1}, v_{2}, v_{3}, v_{4}, v_{5}, v_{6}, v_{7}, v_{8}, v_{9}, v_{10}, v_{11}, v_{12}\right]$, satisfying $\mathbf{v} \cdot \mathbf{w}=$ 1 , are given by,

$$
\begin{aligned}
& v_{1}=0, v_{2}=\frac{\sigma_{1} K_{1}}{K_{1} K_{2}+\sigma_{1} \beta_{1}^{*}}, v_{3}=\frac{\beta_{1}^{*} v_{2}}{K_{1}}, v_{4}=0, v_{5}=0, v_{6}=0, \\
& v_{7}=0, v_{8}=0, v_{9}=0, v_{10}=\frac{\left(K_{8} \beta_{4} \eta_{4}+\sigma_{4}\right) v_{2}}{K_{7} K_{8}}, v_{11}=\frac{\beta_{4} v_{2}}{K_{8}}, v_{12}=0 .
\end{aligned}
$$




\section{Computation of bifurcation coefficient $a$}

It can be shown, by computing the associated non-zero partial derivatives of $F(x)$ (evaluated at the DFE $\mathcal{E}_{0}$ ) that

$$
\begin{aligned}
& a=\frac{2 \mu}{\Pi}\left[\frac{\beta_{1}^{*}\left(\eta_{1} K_{2}+\sigma_{1}\right)}{\sigma_{1}}+\frac{\beta_{2}\left(\eta_{2} K_{4}+\sigma_{2}\right)}{\sigma_{2}}\right] \times \\
& \quad\left\{\left[\frac{\theta_{2} \beta_{21}\left(\eta_{21} K_{8}+\sigma_{21}\right)}{\sigma_{21}}+\frac{\theta_{1} \beta_{12}\left(\eta_{12} K_{6}+\sigma_{12}\right)}{\sigma_{12}}\right]-2\left(2+\frac{K_{2}}{\sigma_{1}}+\frac{K_{4}}{\sigma_{2}}+\frac{\gamma_{1}+\gamma_{2}}{\mu}\right)\right\} .
\end{aligned}
$$

\section{Computation of bifurcation coefficient $b$}

Substituting the eigenvectors $\mathbf{v}$ and $\boldsymbol{w}$ and the respective partial derivatives (evaluated at the DFE $\mathcal{E}_{0}$ ) into the expression

$$
b=\sum_{k, i=1}^{12} v_{k} w_{i} \frac{\partial^{2} f_{k}}{\partial x_{i} \partial \beta_{1}^{*}}(0,0)=\frac{\eta_{1} K_{2}+\sigma_{1}}{\sigma_{1}}>0 .
$$

Since the coefficient $b$ is automatically positive, it follows that the model (1) (or its transformed equivalent (29)) will undergo backward bifurcation if the coefficient $a$, given by (32), is positive.

\section{Appendix B: Proof of Lemma 3}

Proof. Consider the the model (1) with strain 1-only (i.e., let $R_{02}<1$, so that strain 2 dies out as in Theorem 4 ). Further, let $\mathcal{R}_{01}>1$ Lyapunov function:

$$
\mathcal{F}=\left(\frac{\eta_{1} K_{2}+\sigma_{1}}{K_{1}}\right) E_{1}+I_{1}
$$

with Lyapunov derivative given by 


$$
\begin{aligned}
\dot{\mathcal{F}} & =\left(\frac{\eta_{1} K_{2}+\sigma_{1}}{K_{1}}\right) \dot{E}_{1}+\dot{I}_{1} \\
& =\left(\frac{\eta_{1} K_{2}+\sigma_{1}}{K_{1}}\right)\left[\frac{\beta_{1}\left(\eta_{1} E_{1}+I_{1}\right)}{N} S-K_{1} E_{1}\right]+\sigma_{1} E_{1}-K_{2} I_{1} \\
& \leq\left(\frac{\eta_{1} K_{2}+\sigma_{1}}{K_{1}}\right)\left[\beta_{1}\left(\eta_{1} E_{1}+I_{1}\right)-K_{1} E_{1}\right]+\sigma_{1} E_{1}-K_{2} I_{1}, \text { since } S \leq N \text { in } \Omega, \\
& =\beta_{1}\left(\frac{\eta_{1} K_{2}+\sigma_{1}}{K_{1}}\right)\left(\eta_{1} E_{1}+I_{1}\right)-\left(\eta_{1} K_{2}+\sigma_{1}\right) E_{1}+\sigma_{1} E_{1}-K_{2} I_{1}, \\
& =K_{2} \mathcal{R}_{01}\left(\eta_{1} E_{1}+I_{1}\right)-\eta_{1} K_{2} E_{1}-K_{2} I_{1}=K_{2} \mathcal{R}_{01}\left(\eta_{1} E_{1}+I_{1}\right)-\left(\eta_{1} E_{1}+I_{1}\right) K_{2}, \\
& =K_{2}\left(\eta_{1} E_{1}+I_{1}\right)\left(\mathcal{R}_{01}-1\right) \leq 0 . \text { if } \mathcal{R}_{01}>1
\end{aligned}
$$

The proof is completed using the same approach as in the proof of Theorem 3 .

\section{Appendix C: Proof of Theorem 7}

Proof. Consider the model (1) with (14), $\lambda_{1}^{* *}=0$ and $\mathcal{R}_{01}<1<\mathcal{R}_{02}$ (so that the associated unique strain 2-only boundary equilibrium of the model exists). Further, consider the following Lyapunov function:

$$
\begin{aligned}
\mathcal{F} & =S-S^{* *}-S^{* *} \ln \left(\frac{S}{S^{* *}}\right)+E_{2}-E_{2}^{* *}-E_{2}^{* *} \ln \left(\frac{E_{2}}{E_{2}^{* *}}\right) \\
& +\left(\frac{K_{3}-\tilde{\beta}_{2} \eta_{2} S^{* *}}{\sigma_{2}}\right)\left[I_{2}-I_{2}^{* *}-I_{2}^{* *} \ln \left(\frac{I_{2}}{I_{2}^{* *}}\right)\right],
\end{aligned}
$$

with Lyapunov derivative,

$$
\dot{\mathcal{F}}=\dot{S}-\frac{S^{* *}}{S} \dot{S}+\dot{E}_{2}-\left(\frac{E_{2}^{* *}}{E_{2}} \dot{E}_{2}+\frac{K_{3}-\tilde{\beta}_{2} \eta_{2} S^{* *}}{\sigma_{2}}\right)\left(\dot{I}_{2}-\frac{I_{2}^{* *}}{I_{2}} \dot{I}_{2}\right),
$$

so that,

$$
\begin{aligned}
\dot{\mathcal{F}} & =\Pi-\lambda_{2} S-\mu S-\frac{S^{* *}}{S}\left(\Pi-\lambda_{2} S-\mu S\right)+\lambda_{2} S-K_{3} E_{2}-\frac{E_{2}^{* *}}{E_{2}}\left(\lambda_{2} S-K_{3} E_{2}\right) \\
& +\left(\frac{K_{3}-\tilde{\beta}_{2} \eta_{2} S^{* *}}{\sigma_{2}}\right)\left[\sigma_{2} E_{2}-K_{4} I_{2}-\frac{I_{2}^{* *}}{I_{2}}\left(\sigma_{2} E_{2}-K_{4} I_{2}\right)\right] .
\end{aligned}
$$


Hence,

$$
\begin{aligned}
\dot{\mathcal{F}} & =\Pi\left(1-\frac{S^{* *}}{S}\right)+\mu S^{* *}\left(1-\frac{S}{S^{* *}}\right)+\tilde{\beta}_{2} S^{* *} I_{2} \\
& -\tilde{\beta}_{2} \eta_{2} S E_{2}^{* *}-\tilde{\beta}_{2} S I_{2} \frac{E_{2}^{* *}}{E_{2}}+K_{3} E_{2}^{* *}-\frac{K_{3} K_{4} I_{2}}{\sigma_{2}}+\frac{\tilde{\beta}_{2} \eta_{2} S^{* *} K_{4} I_{2}}{\sigma_{2}}-K_{3} E_{2} \frac{I_{2}^{* *}}{I_{2}} \\
& +\frac{K_{3} K_{4} I_{2}^{* *}}{\sigma_{2}}+\tilde{\beta}_{2} \eta_{2} S^{* *} E_{2} \frac{I_{2}^{* *}}{I_{2}}-\frac{\tilde{\beta}_{2} \eta_{2} S^{* *} K_{4} I_{2}^{* *}}{\sigma_{2}} .
\end{aligned}
$$

It can be shown from the model (1) with (14) and $\lambda_{2}^{* *}=0$, at endemic steady-state, that

$$
\begin{aligned}
& \Pi=\tilde{\beta}_{2}\left(\eta_{2} E_{2}^{* *}+I_{2}^{* *}\right) S^{* *}+\mu S^{* *} \\
& K_{3} E_{2}^{* *}=\tilde{\beta}_{2}\left(\eta_{2} E_{2}^{* *}+I_{2}^{* *}\right) S^{* *}, \sigma_{2} E_{2}^{* *}=K_{4} I_{2}^{* *}, \gamma_{2} I_{2}^{* *}=\mu R_{2}{ }^{* *}
\end{aligned}
$$

Substituting the relations in (35) into (34) gives

$$
\begin{aligned}
\dot{\mathcal{F}} & =\left[\tilde{\beta}_{2} \eta_{2} S^{* *} E_{2}^{* *}+\tilde{\beta}_{2} S^{* *} I_{2}^{* *}+\mu S^{* *}\right]\left(1-\frac{S^{* *}}{S}\right)+\mu S^{* *}\left(1-\frac{S}{S^{* *}}\right) \\
& -\tilde{\beta}_{2} \eta_{2} S E_{2}^{* *}-\tilde{\beta}_{2} S I_{2} \frac{E_{2}^{* *}}{E_{2}} \tilde{\beta}_{2} \eta_{2} S^{* *} E_{2}^{* *}+\tilde{\beta}_{2} S^{* *} I_{2}^{* *}-\tilde{\beta}_{2} \eta_{2} S^{* *} E_{2}^{* *} \frac{I_{2}}{I_{2}^{* *}} \\
& -\tilde{\beta}_{2} S^{* *} I_{2}^{* *} \frac{E_{2} I_{2}^{* *}}{E_{2}^{* *} I_{2}}+\tilde{\beta}_{2} S^{* *} I_{2}^{* *},
\end{aligned}
$$

which can be simplified to,

$$
\begin{aligned}
& =\mu S^{* *}\left(2-\frac{S^{* *}}{S}-\frac{S}{S^{* *}}\right)+\tilde{\beta}_{2} \eta_{2} S^{* *} E_{2}^{* *}\left(2-\frac{S^{* *}}{S}-\frac{S}{S^{* *}}\right) \\
& +\tilde{\beta}_{2} S^{* *} I_{2}^{* *}\left(3-\frac{S^{* *}}{S}-\frac{E_{2} I_{2}^{* *}}{E_{2}^{* *} I_{2}}-\frac{I_{2} E_{2}^{* *} S}{I_{2}^{* *} E_{2} S^{* *}}\right) .
\end{aligned}
$$

Finally, since the arithmetic mean exceeds the geometric mean, the following inequalities from (17) hold:

$$
2-\frac{S^{* *}}{S}-\frac{S}{S^{* *}} \leq 0, \quad 3-\frac{S^{* *}}{S}-\frac{E_{2} I_{2}^{* *}}{E_{2}^{* *} I_{2}}-\frac{I_{2} E_{2}^{* *} S}{I_{2}^{* *} E_{2} S^{* *}} \leq 0 .
$$

The proof is completed using the same approach as in the proof of Theorem 3 . 


\section{References}

[1] B. Adams and M. Boots (2007). The influence of immune cross-reaction on phase structure in resonant solutions of a multi-strain seasonal SIR model. Journal of Theoretical Biology. 248: 202-211.

[2] B. Adams and S. Akira (2007). Cross-immunity, invasion and co-existence of pathogen strains in epidemiological models with one-dimensional antigenic space. Mathematical Biosciences. 210(2): 680-699.

[3] R. M. Anderson and R. M. May. Infectious Diseases of Humans: Dynamics and Control. Oxford University Press, Second Edition, 1991.

[4] R. M. Anderson and R. M. May. Population Biology of Infectious Diseases, Springer-Verlag, Berlin, Heilderberg, New York, 1982.

[5] A. S. Ackleh (2005). Competitive exclusion in SIS and SIR epidemic models with total cross immunity and density-dependent host mortality. Discrete and Continuous Dynamical Systems-series B. 5(2): 175-188.

[6] S. Bansal, B. Pourbohloul, N. Hupert, B. Grenfell, L. A. Meyers (2010). The shifting of demographic landscape of pandemic influenza. PLoS ONE. 5(2): e9360. doi:10.1371/journal.pone.0009360.

[7] E. Beretta, Y. Takeuchi (1988). Global stability of Lotka-Volterra diffusion models with continuous time delay. SIAM J. Appl. Math. 48(3): 627-651.

[8] H. J. Bremermann and H.R. Thieme (1989). A competitive exclusion principle for pathogen virulence. J. Math. Biol. 27: 179-190.

[9] F. Brauer (2009). Heterogeneity in disease transmission model. www.cdm.yorku.ca/hetero.pdf. (Accessed September 2012).

[10] J. Carr (1981). Applications Centre Manifold Theory. Springer-Verlag, New York.

[11] C. Castillo-Chavez, H. Wenzhang and L. Jia (1999). Competitive exclusion and co-existence of multiple strains in an SIS STD model. SIAM Journal on Applied Mathematics. 5: 1790-1811.

[12] C. Castillo-Chavez, H. Hethcote, V. Andreason, S. A. Levin and W. Liu (1989). Epidemiolgical models with age sturucture, proportionate mixing, and crossimmunity. Journal of Mathematical Biology. 27: 233-258.

[13] C. Castillo-Chavez and B. Song (2004). Dynamical models of tuberculosis and their applications. Math. Biosci. Engrg. 2: 361-404. 
[14] Centers for Disease Control and Prevention. www.cdc.gov/flu/about/disease/spread.htm (Accessed September 2012).

[15] F. Chamchod, N. F. Britton (2012). On the Dynamics of a two-strain influenza model with isolation. Math. Model. Nat. Phenom. 7(3): 49-61.

[16] G. Chowell, C. E. Ammonb, N.W. Hengartnera and J.M. Hyman (2006). Transmission dynamics of the great influenza pandemic of 1918 in Geneva, Switzerland: Assessing the effects of hypothetical interventions. Journal of Theoretical Biology. 241(2): 193-204.

[17] G. Chowell, L. M. A. Bettencourt, N. Johnson, W. J. Alonso and C. Viboud (2008). The 1918-1919 influenza pandemic in England and Wales: spatial patterns in transmissibility and mortality impact. Proc. R. Soc. B. 275. 501-509.

[18] B. J. Coburn, B. G. Wagner and S. Blower (2009). Modeling influenza epidemics and pandemics: insights into the future of swine flu (H1N1). BMC Medicine.7: 30 .

[19] L. Esteva and C. Vargas (2003). Co-existence of different serotypes of dengue virus, Journal of Mathematical Biology. 46: 31-47.

[20] Z. Feng and X. Jorge Velasco-Hernandez (1997). Competitive exclusion in a vectorhost model for the dengue fever. Journal of Mathematical Biology. 35: 523-544.

[21] N. M. Ferguson, D. A. Cummings, C. Fraser, J. C. Cajka, P. C. Cooley and D. S. Burke (2006). Strategies for mitigating an influenza pandemic. Nature. 442: 448-452.

[22] S. M. Garba and A. B. Gumel (2010). Mathematical recipe for HIV elimination in Nigeria. Journal of the Nigerian Mathematical Society. 29: 51-112.

[23] S. M. Garba and A. B. Gumel (2007). Effect of cross-immunity on the transmission dynamics of two strains of dengue. International Journal of Computer Mathematics. 87(10): 2361-2384.

[24] S. M. Garba, A. B. Gumel and J. M.-S. Lubuma (2011). Dynamically-consistent non-standard finite difference method for an epidemic model. Mathematics and Computer Modelling. 53: 131-150.

[25] L. V. Gubareva, D. V. Novikov and F. G. Hayden (2002). Assessment of hemagglutinin sequence heterogeneity during influenza virus transmission in families. The Journal of Infectious Diseases. 186: 1575-1581. 
[26] A. B. Gumel (2009). Global dynamics of a two strain avian influenza model. Int. j. Comput. Math. 86(1): 85-108.

[27] H. W. Hethcote (2000). The mathematics of infectious diseases. SIAM Rev. 42: 599-653.

[28] D. Y. Melesse and A. B. Gumel (2010). Global asymptotic properties of an SEIRS model with multiple infectious stages. J. Math. Anal. Appl. 366: 202-217.

[29] M. Nuno, Z. Feng, M. Martcheva and C. Castillo-Chavez (2005). Dynamics of twostrain influenza with isolation and partial cross-immunity. SIAM J. Appl. Math. 65(3): 964-982.

[30] M. Iran, M. T. Malik and S. M. Garba (2011). Deterministic model for the role of antivirals in controlling the spread of the H1N1 influenza pandemic. Electronic Journal of Differential Equations. 2011: 1-21.

[31] X. Jorge Velasco-Hernandez (1994). A model for chagas disease involving transmission by vectors and blood transfusion. Theoretical Population Biology. 46: 1-31.

[32] V. Lakshmikantham, S. Leela and A. A. Martynyuk. Stability Analysis of Nonlinear Systems, Marcel Dekker, Inc., New York and Basel, 1989.

[33] J. P. LaSalle (1976). The stability of Dynamical Systems. Regional Conference Series in Applied Mathematics, SIAM, Philadelphia.

[34] X. Li and J. Liu (2010). An age-structured two-strain epidemic model with superinfection. Math. Biosci. Engrg. 7(1): 123-147.

[35] H. Nishiura, (2007). Time variations in the transmissibility of pandemic influenza in Prussia, Germany, from 1918-19. Theor. Biol. Med. Model. 4:20. doi:10.1186/1742-4682-4-20.

[36] H. Nishiura, G. Chowell, M. Safan, C. Castillo-Chavez (2010). Pros and cons of estimating the reproduction number from early epidemic growth rate of influenza A (H1N1) 2009. Theoretical Biology and Medical Modelling. 7: 1.

[37] M. A. Safi and A. B. Gumel (2011). Mathematical analysis of a disease transmission model with quarantine, isolation and an imperfect vaccine. Computer and Mathematics with Application. 61: 3044-3070.

[38] G. Scalia-Tomba (2005). Epidemic model, Multi-strain. Encyclopedia of Biostatistics. http://onlinelibrary.wiley.com/doi/10.1002/0470011815.b2a07017/full (Accessed November 2011). 
[39] O. Sharomi and A. B. Gumel (2008). Dynamical analysis of a multi-strain Model of HIV in the presence of anti-retroviral drugs. Journal of Biological Dynamics. 2(3): 323-345.

[40] O. Sharomi and A. B. Gumel (2009). Re-infection-induced backward bifurcation in the transmission dynamics of chlamydia trachomatis. Journal of Mathematical Analysis and Applications. 356: 96-118.

[41] P. van-den Driessche and J. Watmough, (2002). Reproduction numbers and subthreshold endemic equilibria for compartmental models of disease transmission. Math. Biosci. 180: 29-48.

[42] Y. Yang and X. Fu (2009). Epidemic Propagation dynamics on complex network. Perspectives in Mathematical Sciences. 9: 71-92. 
Table 1: Description of variables and parameters of the model (1)

\begin{tabular}{ll}
\hline \hline Variable & Interpretation \\
\hline$S$ & Population of susceptible individuals \\
$E_{i}(i=1,2)$ & Population of individuals exposed to (latently-infected with) strain $i$ \\
$I_{i}$ & Population of infectious (symptomatically-infected) individuals with strain $i$ \\
$R_{i}$ & Population of individuals who recovered from strain $i$ \\
$E_{i j}(i=1,2 ; i \neq j)$ & Population of individuals who recovered from strain $i$ but exposed to strain $j$ \\
$I_{i j}(i=1,2 ; i \neq j)$ & Population of infectious (with symptoms) individuals who recovered from \\
$M$ & strain $i$ and infectious with strain $j$ \\
& Population of individuals who recovered from infection with both strains \\
\hline Parameter & Interpretation \\
\hline & \\
$\Pi$ & Recruitment rate \\
$\beta_{i}, \beta_{i j}$ & Average lifespan \\
$\eta_{i}, \eta_{i j}$ & Transmission rates \\
$\gamma_{i}, \gamma_{i j}$ & Modification parameters \\
$\sigma_{i}$ & Recovery rates \\
$\theta_{i}$ & Progression rate from $E_{i}$ to $I_{i}$ classes \\
$\delta_{i}, \delta_{i j}$ & Modification parameter of reduced infectivity due to cross-immunity \\
$\xi$ & Disease-induced death rates \\
\hline \hline
\end{tabular}

Table 2: Parameter Values

\begin{tabular}{lll}
\hline \hline Parameter & kazaure1 Value & Reference \\
\hline$\Pi$ & 100 days $^{-1}$ & \\
$\xi$ & 0.4 day $^{-1}$ & Assumed \\
$\mu$ & 0.00004 day $^{-1}(1 / \mu=68$ years $)$ & {$[26,29,30]$} \\
$\beta_{1}, \beta_{2}, \beta_{12}, \beta_{21}$ & {$[0.3,1]$ day $^{-1}$} & {$[29]$} \\
$\eta_{1}, \eta_{2}, \eta_{12}, \eta_{21}$ & 0.5 & {$[30]$} \\
$\gamma_{1}, \gamma_{2}, \gamma_{12}, \gamma_{21}$ & 0.1428 day $^{-1}$ & {$[30]$} \\
$\delta_{1}, \delta_{2}, \delta_{12}, \delta_{21}$ & 0.04227 day $^{-1}$ & {$[37]$} \\
$\sigma_{1}, \sigma_{2}, \sigma_{12}, \sigma_{21}$ & 0.5 day $^{-1}$ & {$[30]$} \\
$\theta_{1}, \theta_{2}$ & 0.5 & {$[29]$} \\
& & \\
\hline \hline
\end{tabular}




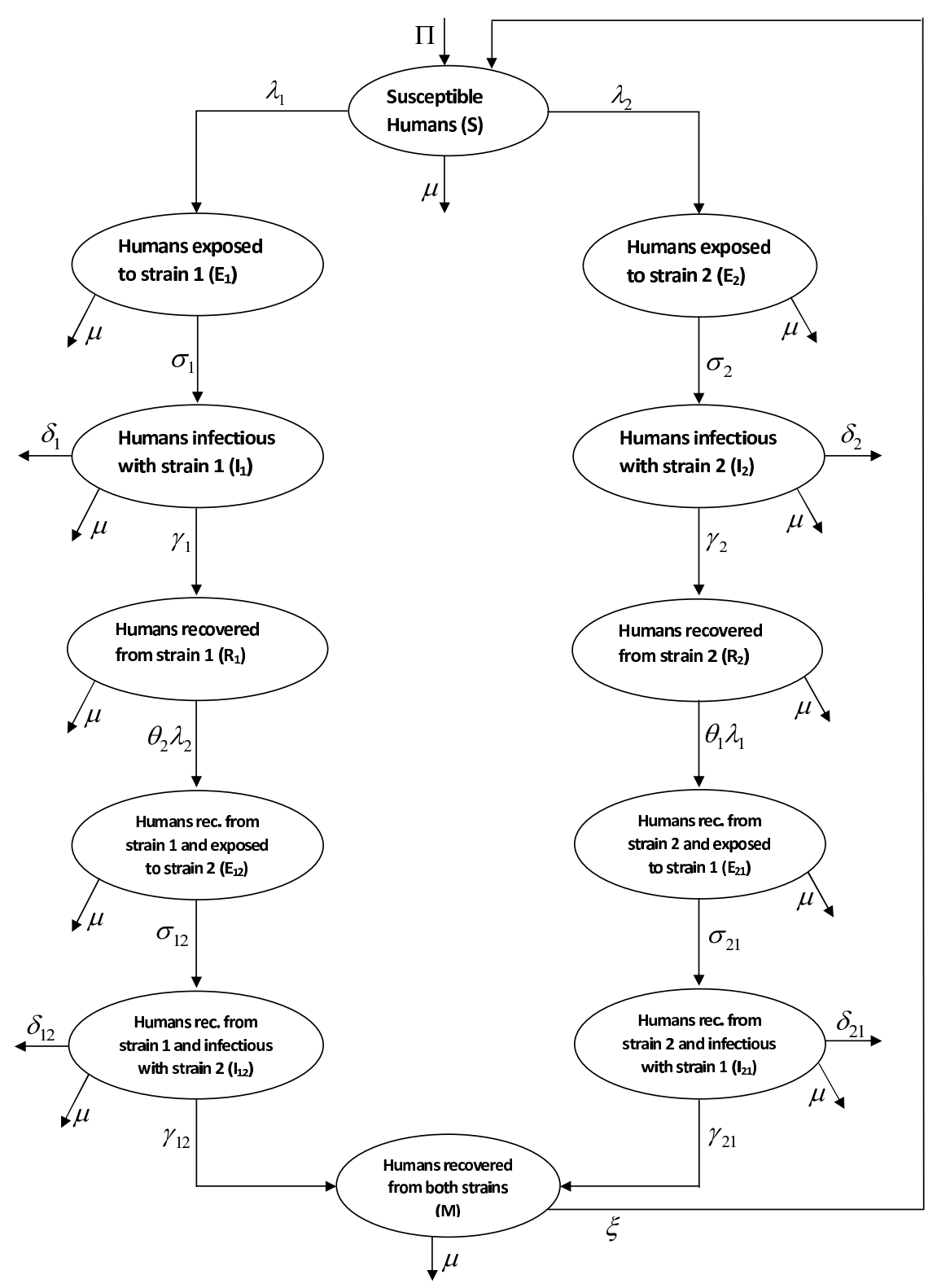

Figure 1: Schematic diagram of the model (1). 

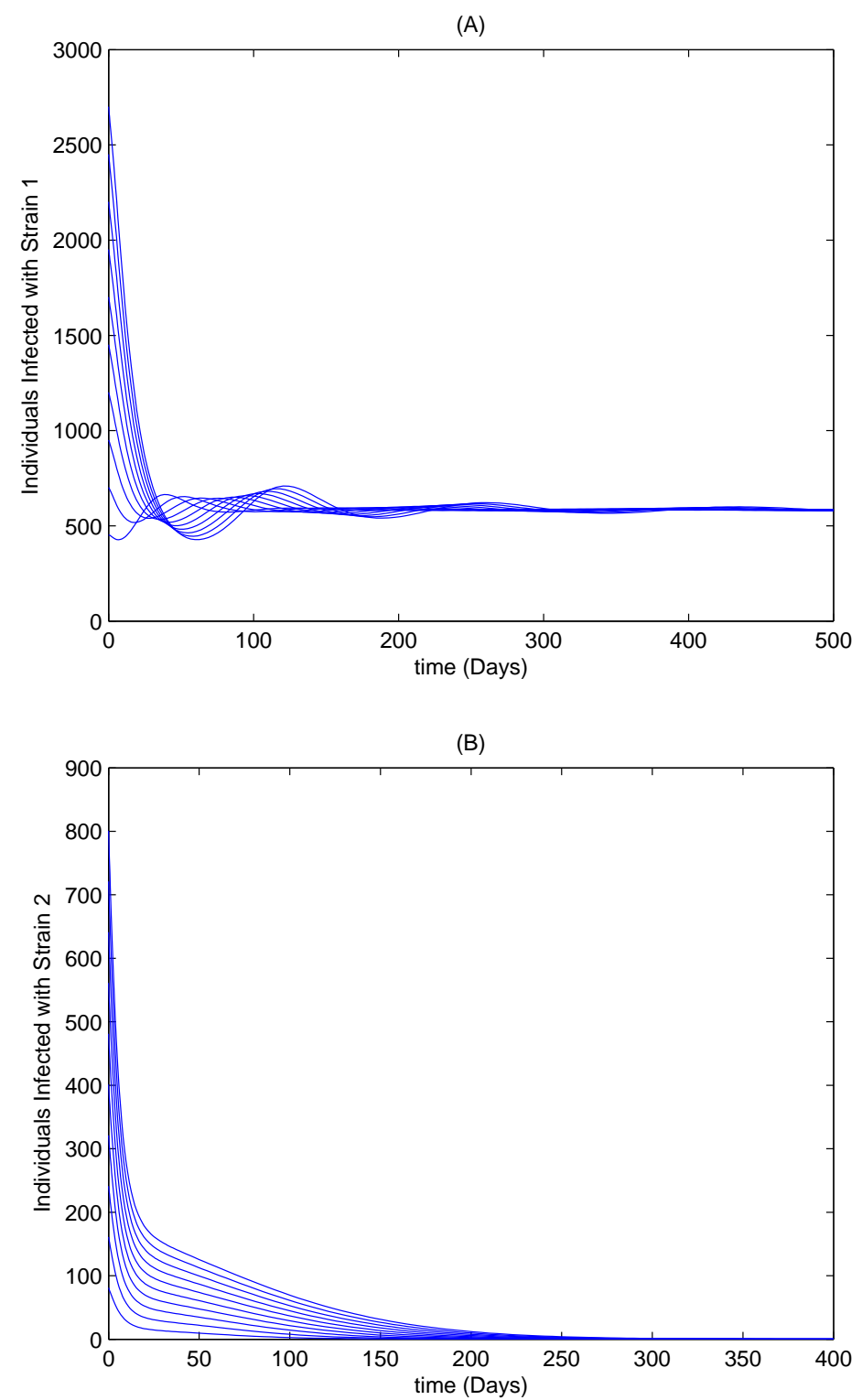

Figure 2: Simulations of the model (1). (A) Population of individuals infected with strain $1\left(E_{1}+I_{1}+E_{21}+I_{21}\right)$; (B) Population of individuals infected with strain 2 $\left(E_{2}+I_{2}+E_{12}+I_{12}\right)$. Parameter values used are: $\beta_{1}=0.6, \beta_{2}=0.1, \beta_{12}=0.3, \beta_{21}=$ 0.5 (so that, $\mathcal{R}_{02}=0.6402<1<\mathcal{R}_{01}=3.8410$ ). Other parameter values used are as given in Table 2. 

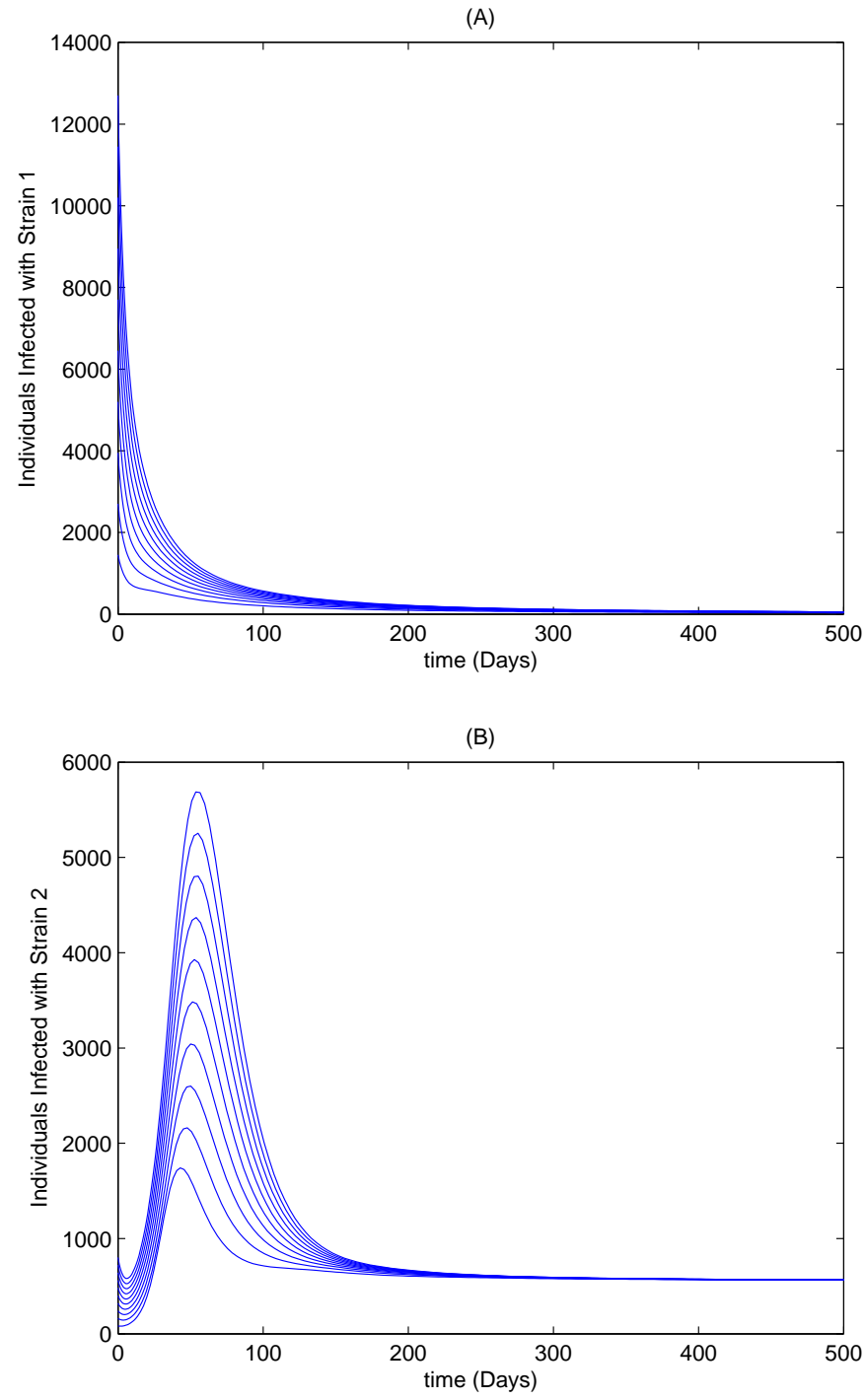

Figure 3: Simulations of the model (1). (A) Population of individuals infected with strain $1\left(E_{1}+I_{1}+E_{21}+I_{21}\right)$; (B) Population of individuals infected with strain 2 $\left(E_{2}+I_{2}+E_{12}+I_{12}\right)$. Parameter values used are: $\beta_{1}=0.1, \beta_{2}=0.6, \beta_{12}=0.5, \beta_{21}=$ 0.5 (so that, $\mathcal{R}_{01}=0.6402<1<\mathcal{R}_{02}=3.2008$ ). Other parameter values used are as given in Table 2. 

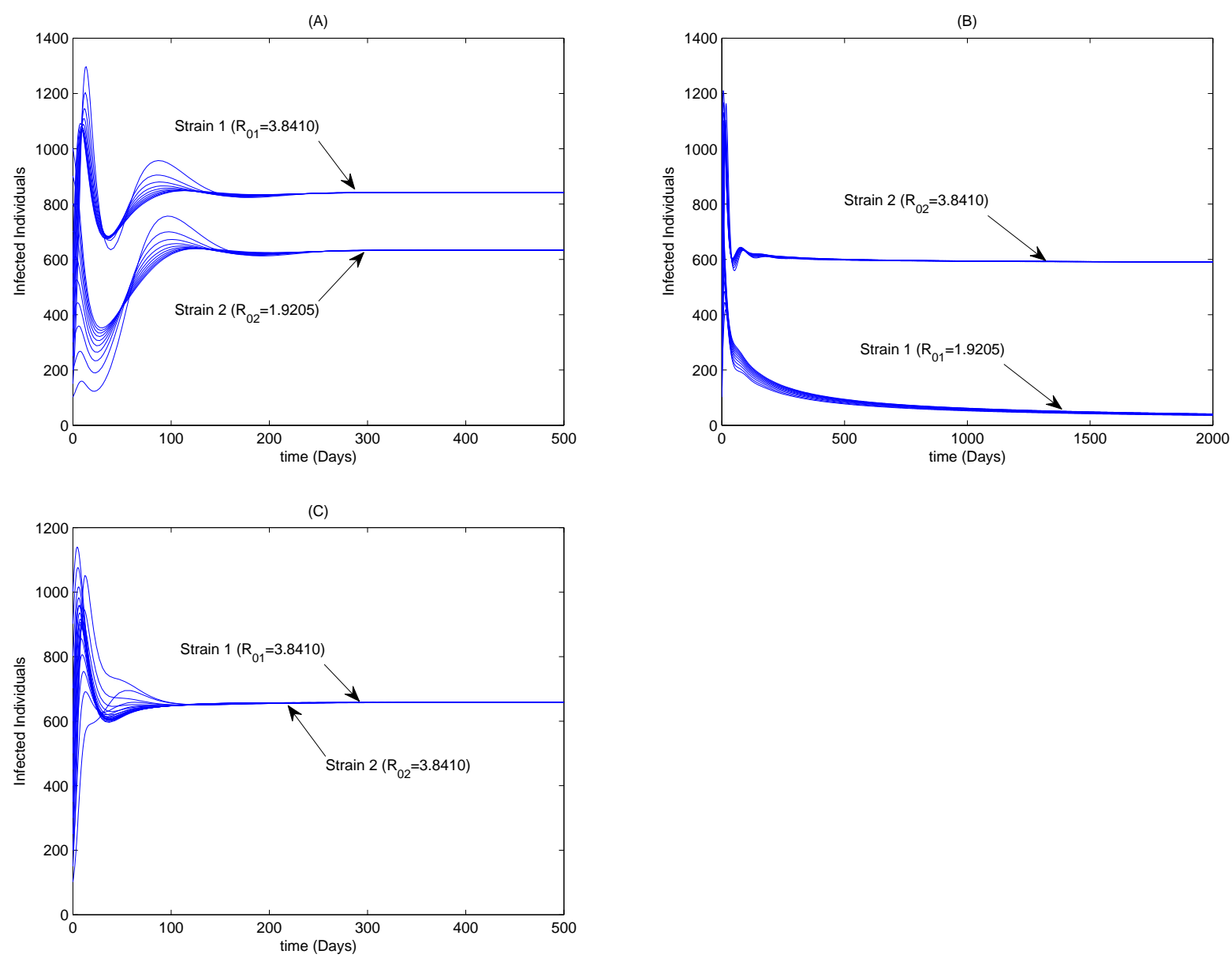

Figure 4: Simulations of model (1) showing co-existence equilibria for: (A) $\mathcal{R}_{01}>$ $\mathcal{R}_{02}>1, \beta_{1}=0.6, \beta_{2}=0.3$ (so that, $\mathcal{R}_{01}=3.8410, \mathcal{R}_{02}=1.9205$ ) (B) $\mathcal{R}_{02}>\mathcal{R}_{01}>$ $1, \beta_{1}=0.3, \beta_{2}=0.6, \beta_{12}=0.5, \beta_{21}=0.5, \quad\left(\right.$ so that, $\mathcal{R}_{02}=3.8410, \mathcal{R}_{01}=1.9205$ ) (C) $\mathcal{R}_{01}=\mathcal{R}_{02}>1, \beta_{1}=0.6, \beta_{2}=0.6$ (so that, $\mathcal{R}_{01}=3.8410, \mathcal{R}_{02}=3.8410$ ). Other parameter values used are as given in Table 2 . 


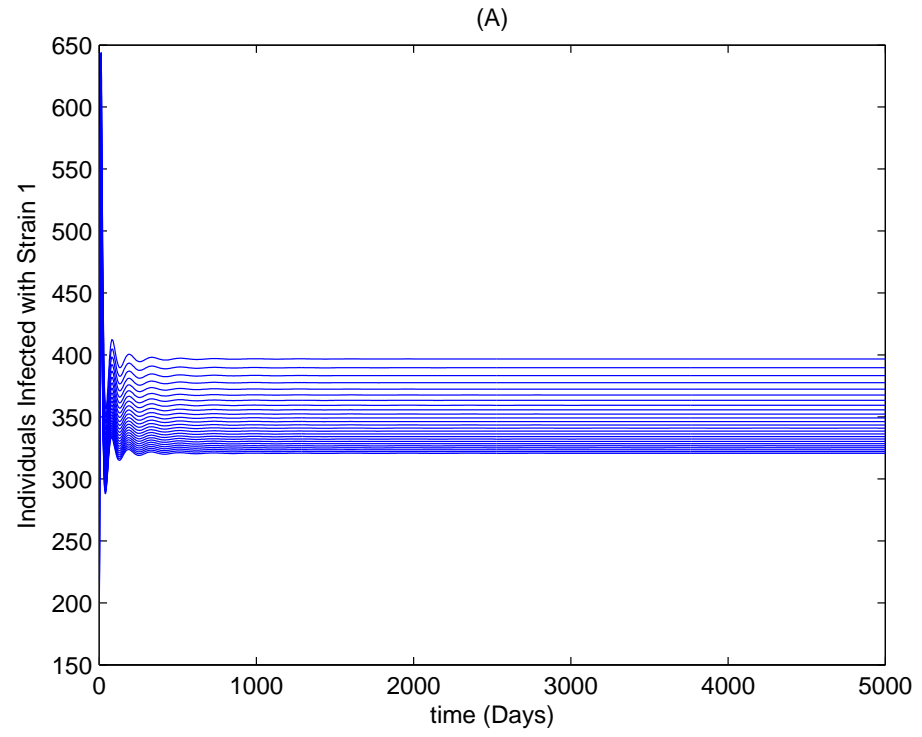

(B)

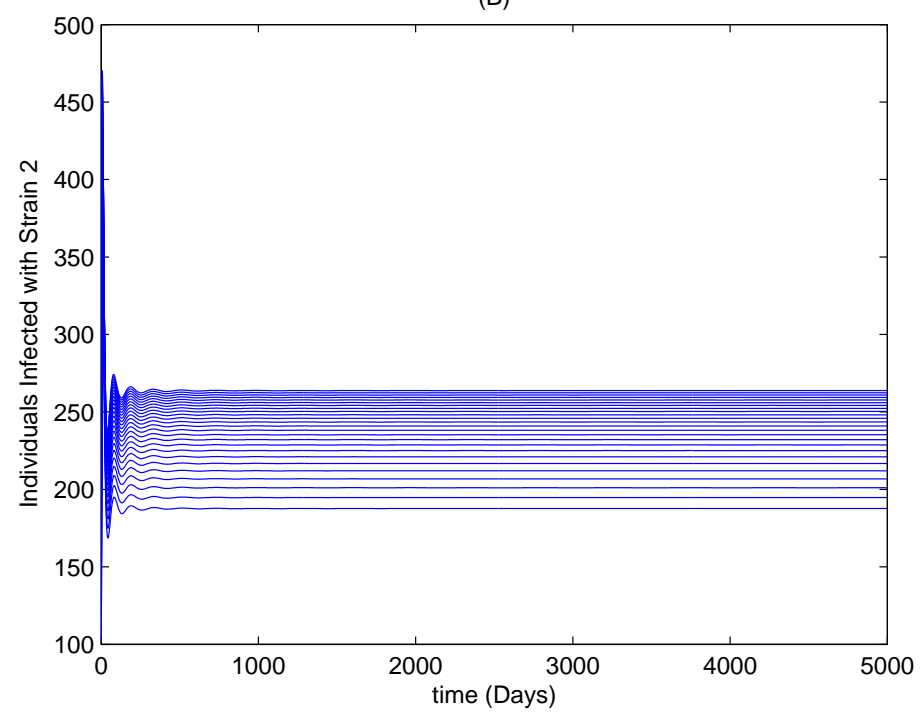

Figure 5: Simulations of the reduced model (7), showing continuum of positive coexistence equilibria for: (A) strain $1\left(E_{1}+I_{1}+E_{21}+I_{21}\right)$; and (B) strain $2\left(E_{2}+I_{2}+\right.$ $\left.E_{12}+I_{12}\right)$. Parameter values used are: $\beta_{1}=0.6, \beta_{2}=0.6, \beta_{12}=0.5, \beta_{21}=0.5, \theta_{1}=$ $0, \theta_{2}=0$ ( so that, $\mathcal{R}_{01}=\mathcal{R}_{02}=3.8410$ ). Other parameter values used are as given in Table 2. 